\title{
Making the Anscombe-Aumann approach to ambiguity suitable for descriptive applications
}

\author{
Stefan Trautmann ${ }^{1}$ - Peter P. Wakker ${ }^{2}$
}

Published online: 28 March 2018

(C) The Author(s) 2018

\begin{abstract}
The Anscombe-Aumann (AA) model, originally introduced to give a normative basis to expected utility, is nowadays mostly used for another purpose: to analyze deviations from expected utility due to ambiguity (unknown probabilities). The AA model makes two ancillary assumptions that do not refer to ambiguity: expected utility for risk and backward induction. These assumptions, even if normatively appropriate, fail descriptively. This paper relaxes these ancillary assumptions to avoid the descriptive violations, while maintaining AA's convenient mixture operation. Thus, it becomes possible to test and apply all AA-based ambiguity theories descriptively while avoiding confounds due to violated ancillary assumptions. The resulting tests use only simple stimuli, avoiding noise due to complexity. We demonstrate the latter in a simple experiment where we find that three assumptions about ambiguity, commonly made in AA theories, are violated: reference independence,
\end{abstract}

Han Bleichrodt and Horst Zank made useful comments. An anonymous referee substantially improved the paper.

Electronic supplementary material The online version of this article (https://doi.org/10.1007/s11166-018-9273-7) contains supplementary material, which is available to authorized users.

Peter P. Wakker

Wakker@ese.eur.nl

Stefan Trautmann

trautmann@uni-hd.de

1 Alfred-Weber-Institute for Economics, University of Heidelberg, Bergheimer Str. 58, 69115 Heidelberg, Germany

2 Econometric Institute, Erasmus University Rotterdam, P.O. Box 1738, Rotterdam, 3000 DR, The Netherlands 
universal ambiguity aversion, and weak certainty independence. The second, theoretical, part of the paper accommodates the violations found for the first ambiguity theory in the AA model—Schmeidler's CEU theory-by introducing and axiomatizing a reference dependent generalization. That is, we extend the AA ambiguity model to prospect theory.

Keywords Ambiguity - Reference dependence $\cdot$ Certainty independence $\cdot$ Prospect theory $\cdot$ Loss aversion

\section{JEL Classifications D81 · D03 · C91}

Keynes (1921) and Knight (1921) emphasized the need to develop theories for decision making when probabilities are unknown. This led Savage (1954) and others to provide a behavioral foundation of (subjective) expected utility: if no objective probabilities are available, then subjective probabilities should be used instead. However, Ellsberg (1961) provided two paradoxes showing that Savage's theory fails descriptively, and according to some also normatively (Ellsberg 1961; Cerreia-Vioglio et al. 2011; Gilboa and Schmeidler 1989; Klibanoff et al. 2005). It led to the development of modern ambiguity theories; i.e., decision theories for unknown probabilities that deviate from expected utility.

Anscombe and Aumann (1963; AA henceforth) presented a two-stage model of uncertainty to obtain a simpler foundation of expected utility than Savage's. ${ }^{1}$ Gilboa and Schmeidler (1989) and Schmeidler (1989) showed that the AA two-stage model is well suited for another purpose: to analyze ambiguity theoretically. Since then, the AA model has become the most-used model for this alternative purpose.

The AA model makes two ancillary assumptions-expected utility for risk and backward induction (see Section 1) — that do not concern ambiguity. ${ }^{2}$ These assumptions have been justified on normative grounds but fail descriptively, as many studies have shown (references in Section 1). They are made only to facilitate the theoretical analysis of ambiguity by providing a convenient linear mixture operation. We show how these ancillary assumptions can be relaxed to become descriptively valid while maintaining the mixture operation. We thus make the AA model suited for descriptive purposes while maintaining its analytical power. AA-based theories of ambiguity can then be applied and tested descriptively while avoiding confounds due to violated ancillary assumptions. We call our modification of the AA model the reduced AA (rAA) model.

We demonstrate the applicability of the rAA method in an experiment (Section 3). This experiment is simple but, as we will see, suffices to falsify most current AAbased ambiguity theories, due to reference dependence. The second, theoretical, part of the paper (Section 4 and further) provides a reference dependent generalization of

\footnotetext{
${ }^{1}$ AA used a three-stage model, but one stage is omitted in modern usage. For empirical applications, this omission was justified by Oechssler et al. (2016).

${ }^{2}$ Some papers relaxing these ancillary assumptions are discussed at the end of Section 7.
} 
Schmeidler's (1989) Choquet expected utility to accommodate the empirical violations found in the first part. This result amounts to extending the AA model to cover Tversky and Kahneman's (1992) prospect theory. Unlike the second part of the paper, the first part avoids using advanced theory so as to provide ready tools to test AA theories for experimentalists. The two parts can be read independently, but are joined in this paper to combine a negative empirical finding on some theories with a positive result on a new theory that solves the problems found. We give a one-sentence description of the rAA method at the end of Section 2. A detailed outline of the paper is at the end of the next section.

\section{Background (substantive and ancillary assumptions) and outline}

This section presents a basic version of the AA model so as to motivate the method that we introduce in the next section. A formalized and general version of the AA model will be presented in the theoretical part of the paper, starting in Section 4. Figure 1a depicts a standard "Savage" act for decision under uncertainty. $E_{1}, \ldots, E_{n}$ denote mutually exclusive and exhaustive events. That is, exactly one will obtain, but it is uncertain which one. Following AA, we assume that a horse race takes place with $n$ horses participating, and exactly one will win. Event $E_{i}$ refers to horse $i$ winning. The act yields consequence $x_{i}$ if event $E_{i}$ obtains. We mostly assume that consequences are monetary, although they can be anything. $U\left(x_{i}\right)$ is the utility of consequence $x_{i}$. $V$ denotes a general functional that represents preferences. It is increasing in all its arguments. Savage (1954) considered the case where $V$ gives subjective expected utility. Nowadays, there is much interest in ambiguity theories, where $V$ can be any such theory, e.g., a multiple prior theory. Such theories are also the topic of this paper.

In decision under risk, we assume probabilities to be known. Then choices are between lotteries (probability distributions). Figure $1 \mathrm{~b}$ denotes a lottery yielding $x_{j}$ with probability $p_{j}$. Following AA, we assume that a roulette wheel is spun to generate the probabilities. Besides the expected utility evaluation depicted, many deviating models have been studied (Starmer 2000).

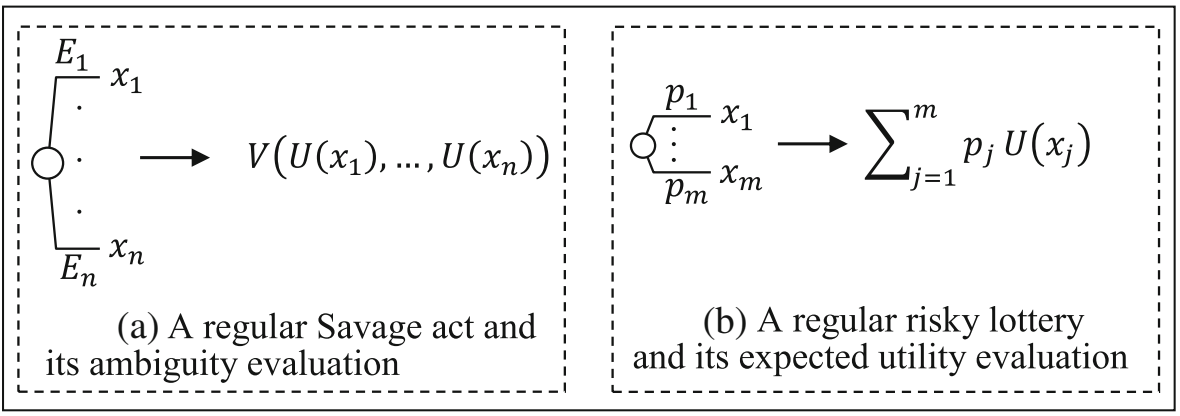

Fig. 1 Traditional (one-stage) choice objects 


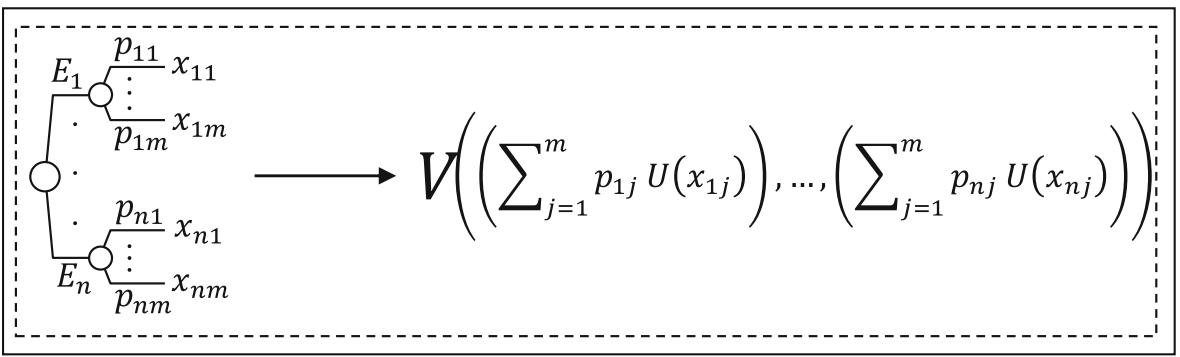

Fig. 2 An Anscombe-Aumann act and its evaluation

Figure 2 depicts an act in AA's model. Both uncertainty and risk are involved. The act is like a Savage act in Fig. 1a, but now consequences are lotteries, i.e., probability distributions over "outcomes" $x_{i j}$. Uncertainty is resolved in two stages. First nature chooses which event $E_{i}$ obtains, resulting in the corresponding lottery. Next the lottery is resolved, resulting in outcome $x_{i j}$ with probability $p_{i j}, j=1, \ldots, m .{ }^{3}$ In AA's model, acts are evaluated as depicted. First, every lottery of the second stage is evaluated by its expected utility. Next, an ambiguity functional $V$ is applied to those expected utilities as it was to utilities in Fig. 1a. The evaluation of the ambiguity by the functional $V$ is of central interest in the modern ambiguity literature. The evaluation of the lotteries only serves to facilitate the analysis of ambiguity in the first stage. The evaluation of each lottery in the second stage is independent of what happens at the other branches in the figure. We can, for instance, replace each lottery by its certainty equivalent derived "in isolation" in Fig. 1b, and then evaluate the resulting ambiguous act as in Fig. 1a. That is, we are using backward induction here.

We list the two assumptions made, and add two more: (1) lotteries, being unambiguous, are evaluated using expected utility (EU); (2) backward induction is used to evaluate the two stages; (3) there is no reference dependence, with gains and losses treated the same; (4) there is universal ambiguity aversion. The last two assumptions concern ambiguity and are, therefore, of central interest. They are called substantive. Assumptions 1 and 2 define the AA model, with its two-stage structure. They only serve to simplify the mathematical analysis and are, therefore, called ancillary.

The purpose of this paper is descriptive. We, therefore, wish to avoid descriptive problems of the ancillary assumptions. As regards the first assumption, Allais' (1953) thought experiment provided the first evidence against EU for risk, later confirmed by many empirical studies. It led to the popular prospect theory (Kahneman and Tversky 1979; Tversky and Kahneman 1992). Surveys of violations of EU for risk include Birnbaum (2008), Edwards (1954), Fehr-Duda and Epper (2012), Fox et al. (2015), Schmidt (2004), Slovic et al. (1988), and Starmer (2000). In view of the many violations of EU found, Assumption (1) is currently considered to be descriptively

\footnotetext{
${ }^{3}$ For simplicity of notation, we often assume that all lotteries in one act have the same number, $m$, of outcomes. This can always be achieved by adding zero probability outcomes to some lotteries.
} 
unsatisfactory. Several authors argued that it is also normatively undesirable (Allais 1953; Machina 1989).

Assumption (2), backward induction, is a kind of monotonicity condition. If we only focus on consequences that are sure money amounts (degenerate lotteries; Fig. 1a), then the condition is uncontroversial. However, it becomes debatable if consequences are nondegenerate lotteries as in Fig. 2. Then the condition implies that the decision maker's evaluation of the lottery faced there, i.e., of the act conditional on the event $E_{i}$ that obtained, is independent of what happens outside of $E_{i}$. This is a form of separability rather than of monotonicity (Bommier 2017 p. 106; Machina 1989 p. 1624), which may be undesirable for ambiguous events $E_{i}$. Although most papers using the AA model do not discuss this assumption explicitly, several recent papers have criticized it (Bommier 2017; Bommier et al. 2017 Footnote 7; Cheridito et al. 2015; Machina 2014 p. 385 3rd bulleted point; Saito 2015; Schneider and Schonger 2017; Skiadas 2013 p. 63; Wakker 2010 Section 10.7.3).

Dynamic optimization principles such as backward induction that are self-evident under expected utility become problematic and cannot all be satisfied under nonexpected utility (Machina 1989). Several authors have therefore argued against backward induction for nonexpected utility on normative grounds. ${ }^{4}$ Many studies have found empirical violations of backward induction. ${ }^{5}$ We conclude that both ancillary assumptions are descriptively problematic and, according to several authors, also normatively problematic. Our rAA model therefore aims to avoid the problems just discussed.

We now turn to a detailed outline of the paper. Section 2 explains the rAA model informally, showing how to test AA theories without being affected by violations of the ancillary assumptions. In particular, no two-stage uncertainty as in Fig. 2 occurs in the rAA model, and we only use stimuli as in Fig. 1. An additional advantage of our stimuli is that they are less complex, reducing the burden for subjects and the noise in the data. Dominiak and Schnedler (2011) and Oechssler et al. (2016) tested Schmeidler's (1989) uncertainty aversion for two-stage acts, and found no clear relations with Ellsberg-type ambiguity aversion. This can be taken as evidence against the descriptive usefulness of two-stage acts.

Section 3 illustrates our approach in a simple experiment. Unsurprisingly, we find that losses are treated differently, with more ambiguity seeking, than gains (reference dependence). We have thus tested and falsified the substantive Assumptions 3 and 4. Many studies have demonstrated reference dependence outside of ambiguity, and several have done so within ambiguity. ${ }^{6}$ Our experiment shows it in a simpler way and is the first to have done so for the AA model. It may be conjectured that AA theories could indirectly model the reference dependence found. This conjecture holds

\footnotetext{
${ }^{4}$ See Dominiak and Lefort (2011), Eichberger and Kelsey (1996), Karni and Schmeidler (1991), Machina (1989), Machina (2014 Example 3), Ozdenoren and Peck (2008), and Siniscalchi (2011).

${ }^{5}$ See Cubitt et al. (1998), Dominiak et al. (2012), and Yechiam et al. (2005).

${ }^{6}$ See Abdellaoui et al. (2005), Baillon and Bleichrodt (2015), de Lara Resende and Wu (2010), Dimmock et al. (2015), Du and Budescu (2005), and Kocher et al. (2018).
} 
true for the smooth model (Klibanoff et al. 2005) and other utility-driven theories of ambiguity. ${ }^{7}$ However, we prove that it does not hold true for most commonly used AA theories, because weak certainty independence, a necessary condition for most theories, ${ }^{8}$ is violated. Baillon and Placido (2017) also tested this condition and also found it violated. Generalizations of these theories are therefore desirable. We turn to those in the next, theoretical, part of the paper, with definitions and basic results in Section 4 and the reference dependent generalization of Schmeidler (1989) in Section 5. Faro (2005, Ch. 3 ) provided an alternative ambiguity model with reference dependence.

Our generalization of Schmeidler's model can accommodate loss aversion, and ambiguity aversion for gains combined with ambiguity seeking for losses, as in prospect theory. In many applications of ambiguity (asset markets, insurance, health) the gain-loss distinction is important, and descriptive models that assume referenceindependent universal ambiguity aversion cannot accommodate this. As regards our finding of violations of weak certainty independence, reference dependence is the only generalization needed to accommodate these violations. Weak certainty independence remains satisfied if we restrict our attention to gains or to losses. Section 6 analyzes loss aversion under ambiguity. A discussion, with implications for existing ambiguity theories, is in Section 7. Section 8 concludes.

A model-theoretic isomorphism of the rAA model with the full AA model is in Appendix E. Its implications can be stated in simple terms for experimentalists, without requiring a study of its formal content: Although the rAA model is a submodel of the full AA model, every ambiguity property that can be defined in the full AA model can be tested in the rAA model using the method explained in the next section. No information on ambiguity is lost by restricting to the rAA model. A simple test such as the one in Section 3 can be devised for every ambiguity condition other than weak certainty independence.

\footnotetext{
${ }^{7}$ See Chew et al. (2008), Kahneman and Tversky (1975 pp. 30-33), Nau (2006), Neilson (2010), and Skiadas' (2015 source-dependent theory). These models still focus on normative universal ambiguity aversion. They cannot model the empirically prevailing ambiguity seeking for unlikely events joint with ambiguity aversion for likely events (Zeckhauser and Viscusi 1990; reviewed by Camerer and Weber 1992, and Trautmann and van de Kuilen 2015), or the kinks in preferences that are often found (Ahn et al. 2014). Dobbs (1991) also proposed a general recursive utility-driven theory of ambiguity and emphasized the importance of different attitudes for gains than for losses, which he demonstrated in an experiment. His approach thus is close to ours. Viscusi and O'Connor (1984) similarly found prevailing ambiguity seeking for losses except when they were unlikely, in which case ambiguity aversion was prevailing.

${ }^{8}$ See Chambers et al. (2014): dispersion aversion; Maccheroni et al. (2006): variational model; Saponara (2017); Siniscalchi (2009): vector theory; several multiple priors theories (Chateauneuf 1991 and Gilboa and Schmeidler 1989: maxmin expected utility; Gajdos et al. 2008: contraction model; Ghirardato et al. 2004, also their $\alpha(f)$ model); Grant and Polak (2013); Jaffray (1994): $\alpha$-maxmin theory; Kopylov (2009): choice deferral; Skiadas (2013): scale-invariant uncertainty aversion; Strzalecki (2011): multiplier preferences. Exceptions are Chateauneuf and Faro (2009), Chew et al. (2008), Hayashi and Miao (2011), Klibanoff et al. (2005), and Skiadas (2013 source-dependent theory). Further, the violation that we found involved only binary acts, implying that every model agreeing with CEU on this subdomain is violated too (Ghirardato and Marinacci 2001: biseparable preference; Luce 2000 Ch. 3: binary rank-dependent utility; tested by Choi et al. 2007).
} 


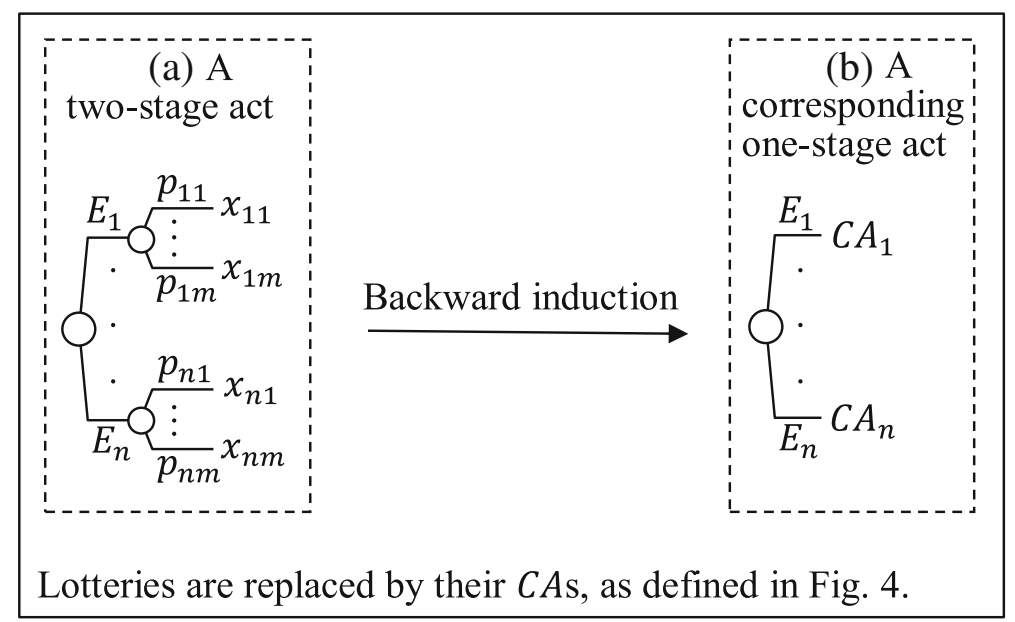

Fig. 3 Relating a general two-stage act of the AA model to a one-stage ("rAA") act

The first, empirical part of this paper, preceding Section 4, makes empirical studies of the AA model possible, providing an easy recipe. It is accessible to readers with no mathematical background. We postpone formal definitions and results to the second, theoretical part, in Section 4 and further. Given the negative finding in the first part, with violations of most existing AA ambiguity theories, the second part presents a positive result: the first reference-dependent AA theory.

\section{The reduced AA model and the AA twin of the decision maker}

This section explains the reduced AA model informally, so that it can easily be used by experimenters. Appendix E gives a formal presentation. Figure 3 a depicts a twostage AA act as in Fig. 2.

We do not use two-stage acts when empirically measuring the preferences of the decision maker. We only consider one-stage acts as: (1) in Fig. 3b, where all secondstage lotteries are degenerate and only uncertainty about the horses matters, or: (2) in Fig. 4, where the first-stage uncertainty, not depicted, is degenerate and only the risks of the roulette wheel matter. In Fig. 4, we avoid degenerate lotteries by only considering lotteries that give the worst outcome, -20 in our case, with a probability of at least 0.2 , and give the best outcome, 10, with a probability of at least 0.2 .

The preference relation of the decision maker over the domain of one-stage acts just described (Figs. 3b and 4) is denoted $\succcurlyeq$. This domain and $\succcurlyeq$ are called the reduced AA (rAA) model. We assume that EU (expected utility) holds for risky choices $\succcurlyeq$ in the rAA domain. Most violations of EU occur when tails of distributions are relevant, but on the RAA domain the tails are fixed and play no role. Hence, EU is empirically plausible here, and we assume it. Further explanation and references are in Section 7. As for the ancillary assumption of backward induction, it is vacuous on the rAA domain. 


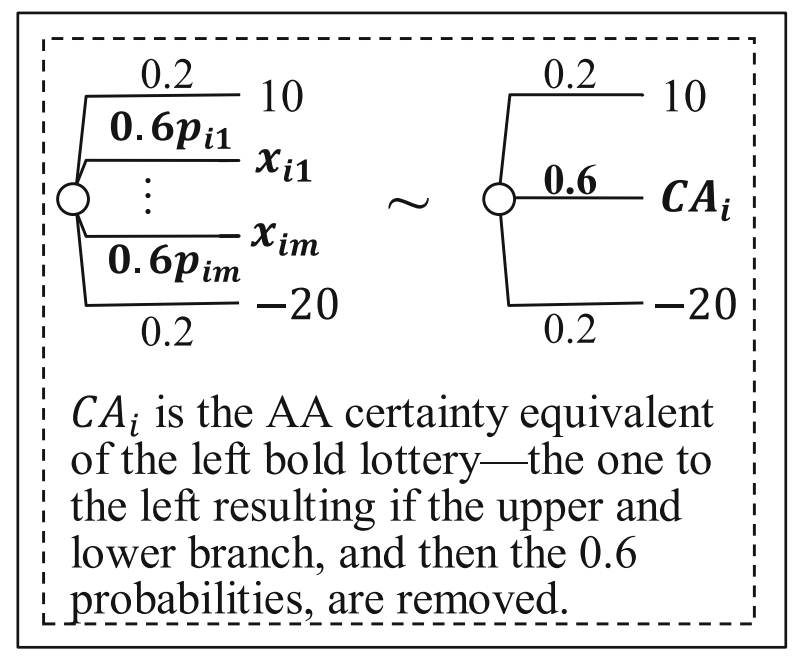

Fig. 4 Defining a conditional certainty equivalent

In theoretical analyses of the AA model, two-stage acts do play a role. To capture them in our rAA method, we do not consider the actual preferences of the decision maker over them, but instead we consider a preference relation $\succcurlyeq *$ of what we call the AA twin of the decision maker. The asterisk indicates that these preferences do not need to agree with the actual empirical preferences of the decision maker, but belong to her idealized AA twin. This $\succcurlyeq *$ agrees with $\succcurlyeq$ on the rAA domain, but extends it to the whole AA model, and is required to satisfy the AA conditions (EU for risk and backward induction). As we explain next, $\succcurlyeq^{*}$ exists and is uniquely determined this way.

Consider Fig. 4. Because the stimuli come from the rAA domain, the indifference also holds for $\sim^{*}$ instead of $\sim$. Because $\succcurlyeq^{*}$ satisfies EU, the $\sim^{*}$ indifference is maintained if we remove the "common-consequence" upper and lower 0.2 branches, and then the "common-ratio" 0.6 probabilities. That is, for each $i, C A_{i}$ for sure is $\sim^{*}$ equivalent to the lottery at branch $E_{i}$ in Fig. 3a:

$$
C A_{i} \sim^{*}\left(p_{i 1}: x_{i 1}, \ldots, p_{i m}: x_{i m}\right)
$$

using the obvious notation for lotteries. By backward induction (CE substitution), the act in Fig. 3a is $\sim^{*}$ indifferent to the act in Fig. 3b, which is again in the rAA domain governed by $\succcurlyeq$. This way, the $\sim^{*}$ indifference class of every two-stage AA act is uniquely determined and, hence, so is $\succcurlyeq^{*}$. We can infer the whole relation $\succcurlyeq^{*}$ this way. We summarize the procedure, for any preference relationship $\succcurlyeq^{*}$ :

(1) Every act from rAA is left unaltered because $\succcurlyeq^{*}$ agrees with $\succcurlyeq$ on the rAA domain.

(2) For every lottery, its CA certainty equivalent is defined through Eq. 1 and Fig. 4.

(3) Every two-stage act is replaced by a one-stage act as in Fig. 3. 
Point (2) means that $C A$ s are $\succcurlyeq^{*}$ certainty equivalents. Stating the rAA method in one sentence:

We can find out any AA preference $\succcurlyeq *$ from rAA preferences $\succcurlyeq$ by using the substitution in Fig. 3.

We can thus apply all techniques from the AA model to analyze $\succcurlyeq^{*}$ and infer properties of the uncertainty attitude of $\succcurlyeq *$ on horse acts using only $\succcurlyeq$ preferences on the rAA domain as empirical inputs. The uncertainty attitude-which may deviate from subjective expected utility-of the AA twin $\succcurlyeq *$ is identical to that of $\succcurlyeq$. Thus, all results from the AA literature immediately apply to $\succcurlyeq$.

In applications, if only few CAs are to be measured, then we can measure each one separately as in Fig. 4. If there are many, we can carry out a few measurements as in Fig. 4, derive the EU utility function from them, and use it to determine all CAs that we need. Two drawbacks of the rAA method must be acknowledged. First, the stimuli used for measuring risk attitudes in Fig. 4 are made more complex by the mixing in of the best and worst outcomes. Second, when testing mixture conditions from the full AA model, we have to modify every two-stage act into an rAA act as just described.

The following section gives an illustration of the rAA method, showing how it can be used to test AA theories experimentally. We test weak certainty independence there, a preference condition necessary for many AA theories.

\section{Experimental illustration of the reduced AA model and reference dependence}

This section demonstrates the rAA model in a small experiment. First, we present a common example. The unit of payment in the example can be taken to be money or utility. In the experiment that follows, the unit of payment will be utility and not money, so that the violations found there directly pertain to the general AA model. Because the rAA model is a submodel of the full AA model (but large enough to recover the latter entirely), any violation of a preference condition found from $\succcurlyeq$ in the rAA model immediately gives a violation of that preference condition for $\succcurlyeq *$ in the full AA model.

Example 1 (Reflection of ambiguity attitudes) A known urn K contains 50 red (R) and 50 black (B) balls. An unknown (ambiguous) urn A contains 100 black and red balls in unknown proportion. One ball will be drawn at random from each urn, and its color will be inspected. $R_{k}$ denotes the event of a red ball drawn from the known urn, and $B_{k}, R_{a}$, and $B_{a}$ are analogous. People usually prefer to receive $€ 10$ under $B_{k}$ (and 0 otherwise) rather than under $B_{a}$ and they also prefer to receive $€ 10$ under $R_{k}$ rather than under $R_{a}$. These choices reveal ambiguity aversion for gains.

We next multiply all outcomes by -1 , turning them into losses. This change of sign can affect decision attitudes. Many people now prefer to lose $€ 10$ under $B_{a}$ rather than under $B_{k}$ and also to lose $€ 10$ under $R_{a}$ rather than under $R_{k}$. That is, many people exhibit ambiguity seeking for losses. 
The above example illustrates that ambiguity attitudes are different for gains than for losses, making it desirable to separate these, similar to what has been found for risk (Tversky and Kahneman 1992). This separation is impossible in most current ambiguity theories. We tested the above choices in our experiment. Subjects were $N=45$ undergraduate students from Tilburg University. We asked both for preferences with red as the winning color and for preferences with black as the winning color. This way we avoided suspicion about the experimenter rigging the composition of the unknown urn (Pulford 2009).

We scaled utility to be 0 at 0 and 10 at $€ 10$. That is, the winning amount was always $€ 10$. We wanted the loss outcome to be -10 in utility units for each subject, which required a different monetary outcome $\alpha$ for each subject. Thus, under EU as assumed in the AA model and as holding for the AA twins of the subjects, we must have, with the usual notation for lotteries (probability distributions over money),

$$
€ 0 \sim^{*}(0.5: € 10,0.5: € \alpha) \text {. }
$$

One simplifying notation for lotteries: we often rewrite $(p: \alpha, 1-p: \beta)$ as $\alpha_{p} \beta$. The indifference displayed involves a degenerate (nonrisky) prospect ( $€ 0$ ), and those are known to cause many violations of the assumed EU. ${ }^{9}$ We therefore use the modification in Fig. 4. We write $R=\left(€ 10_{0.5}(-€ 20)\right)$, and rather elicit the following indifference from our subjects, as in Fig. 4, using the common probabilistic mixtures of lotteries, and mixing in $R$ with weight 0.4 :

$$
\left(R_{0.4} € 0\right) \sim\left(R_{0.4}\left(€ 10_{0.5} € \alpha\right)\right) .
$$

Under EU as holding for the AA twin, the latter indifference also holds for $\sim^{*}$ and is equivalent to the former, but the latter indifference is less prone to violations of EU, so that our subjects agree with their AA twins here.

To elicit the indifference in Eq. 3 from each subject, we asked each subject to choose between lotteries (replacing $\alpha$ in Eq. 3 by $-j$ ),

$(0.2: € 10,0.6: € 0,0.2:-€ 20)$ ("safe") and $(0.2: € 10,0.3: € 10,0.3:-€ j, 0.2:-€ 20)$ ("risky") for each $j=0,2,4, \ldots, 18,20$. If the subject switched from risky to safe between $-j$ and $-j-2$, we defined $\alpha$ to be the midpoint between these two values, i.e., $\alpha=-j-1$. We then assumed indifference between the safe and risky prospect with that outcome $\alpha$ instead of $-j$ in the risky prospect. We used the monetary outcome $\alpha$, depending on the subject, as the loss outcome for this subject. This way the loss outcome was -10 in utility units for each subject (as for their AA twin). ${ }^{10}$ Details of the experiment are in the Online Appendix.

We elicited the preferences of Example 1 from our subjects using utility units, with the gain outcome $€ 10$ giving utility +10 , and the loss outcome $\alpha$ giving utility -10 . Combining the bets on the two colors, the number of ambiguity averse choices was larger for gains than for losses (1.49 vs. 1.20, $z=2.01, p<.05$, Wilcoxon test, two-sided), showing that ambiguity attitudes are different for gains than for losses. We replicate strong ambiguity aversion $(z=3.77, p<.01$, Wilcoxon test, twosided) for gains, but we cannot reject the null of ambiguity neutrality $(z=1.57, p>$

\footnotetext{
${ }^{9}$ See Bruhin et al. (2010), Chateauneuf et al. (2007), and McCord and de Neufville (1986).

${ }^{10}$ Section 6 discusses how our measurement of utility incorporates loss aversion under risk.
} 
.10 , Wilcoxon test, two-sided) for losses. ${ }^{11}$ Our experiment confirms that attitudes towards ambiguity are different for gains than for losses, suggesting violations of most ambiguity models used today. The following sections will formalize this claim.

\section{Definitions, notation, classical expected utility, and Choquet expected utility for mixture spaces}

This section provides definitions and well-known results. Proofs are in Ryan (2009). We present our main theorems for general mixture spaces, which covers the traditional two-stage AA model, our rAA model, and also some other models. By Observation 5 in the Appendix, all results proved in the literature for the traditional two-stage AA model also hold for general mixture spaces. $M$ denotes a set of $c o n-$ sequences, with generic elements $x, y . M$ is a mixture space: it is endowed with a mixture operation $x_{p} y: M \times[0,1] \times M \rightarrow M$, also denoted $p x+(1-p) y$, satisfying (i) $x_{1} y=x$ [identity]; (ii) $x_{p} y=y_{1-p} x$ [commutativity]; (iii) $\left(x_{p} y\right)_{q} y=x_{p q} y$ [associativity]. The first example below was popularized by Schmeidler (1989) and Gilboa and Schmeidler (1989).

Example 2 (Two-stage AA model) $D$ denotes a set of (deterministic) outcomes, and $M$ consists of all (roulette) lotteries, which are probability distributions over $D$ taking finitely many values. The mixture operation concerns probabilistic mixing.

Example $3 M=I R$ and mixing is the natural mixing of real numbers.

Our rAA model provides another example (Appendix E). $S$ denotes the state space. It is endowed with an algebra of subsets, called events. An algebra contains $S$ and $\emptyset$ and is closed under complementation and finite unions and intersections. An act $f=\left(E_{1}: f_{1}, \ldots, E_{n}: f_{n}\right)$ takes values $f_{i}$ in $M$ and the $E_{i}$ 's are events partitioning the state space. The set of acts, denoted $\mathcal{A}$, is endowed with pointwise mixing, which satisfies all conditions for mixture operations. Hence, $\mathcal{A}$ itself is also a mixture space. A constant act $f$ assigns the same consequence $f(s)=x$ to all $s$. It is identified with this consequence.

Preferences are over the set of acts $\mathcal{A}$ and are denoted $\succcurlyeq$, inducing preferences $\succcurlyeq$ over consequences through constant acts. Strict preference $\succ$ and indifference $\sim$ are defined as usual. A function $V$ represents $\succcurlyeq$ if $V: \mathcal{A} \rightarrow I R$ and $f \succcurlyeq g \Leftrightarrow V(f) \geq$ $V(g)$. If a representing function exists then $\succcurlyeq$ is a weak order, i.e., $\succcurlyeq$ is complete (for all acts $f$ and $g, f \succcurlyeq g$ or $g \succcurlyeq f$ ) and transitive. $\succcurlyeq$ is nontrivial if (not $f \sim g$ ) for some $f$ and $g$ in $\mathcal{A}$.

Continuity holds if, whenever $f \succ g$ and $g \succ h$, there are $p$ and $q$ in $(0,1)$ such that $f_{p} h \succ g$ and $f_{q} h \prec g$. Hence, continuity relates to the mixing of consequences

\footnotetext{
${ }^{11}$ Testing is against the null of one ambiguity averse choice in two choice situations. The exact distribution of subjects choosing the ambiguous option never, once, or twice is $(28,11,6)$ for gains, and $(21,12,12)$ for losses.
} 
and does not refer to variations in states of nature. In the two-stage AA model, continuity relates to probability (as part of consequences). An affine function $u$ on $M$ satisfies $u\left(x_{p} y\right)=p u(x)+(1-p) u(y)$. In the two-stage AA model, a function is affine if and only if it is EU (defined in Appendix E; it follows from substitution and induction).

Monotonicity holds if $f \succcurlyeq g$ whenever $f(s) \succcurlyeq g(s)$ for all $s$ in $S$. It is nontrivial if the $f(s)$ 's are nondegenerate lotteries as in Example 2. Monotonicity then implies that the decision maker's evaluation of $f(s)$, i.e., of $f$ conditional on state $s$, is independent of what happens outside of $s$. It was discussed in Section 1.

The following condition is the most important one in the axiomatization of affine representations and, hence, of EU.

Definition 1 Independence holds on $M$ if

$$
x \succ y \Rightarrow x_{p} c \succ y_{p} c
$$

for all $0<p<1$ and consequences $x, y$, and $c$.

Theorem 1 (von Neumann-Morgenstern) The following two statements are equivalent:

(i) There exists an affine representation $u$ on the consequence space $M$.

(ii) The preference relation $\succcurlyeq$ when restricted to $M$ satisfies the following three conditions: (a) weak ordering; (b) continuity; (c) independence.

In (i), $u$ is unique up to level and unit.

Uniqueness of $u$ up to level and unit means that another function $u^{*}$ satisfies the same conditions as $u$ if and only if $u^{*}=\tau+\sigma u$ for some real $\tau$ and positive $\sigma$. Affinity, independence, and Theorem 1 can be applied to any mixture set other than $M$, such as the set of acts $\mathcal{A}$. Formally, our term $A A$ model refers to Example 2 plus the preference conditions considered so far in this section, being weak ordering, continuity, monotonicity, and independence on $M$, implying an affine (i.e., EU) representation on $M$. It is a two-stage model. It does not further restrict ambiguity attitudes, i.e., the preference relation over acts, and is assumed in most papers on ambiguity nowadays. We now turn to two classic results.

Anscombe and Aumann's subjective expected utility. A probability measure $P$ on $S$ maps the events to $[0,1]$ such that $P(\emptyset)=0, P(S)=1$, and $P$ is additive $(P(E \cup$ $F)=P(E)+P(F)$ for all disjoint events $E$ and $F)$. Subjective expected utility $(S E U)$ holds if there exists a probability measure $P$ on $S$ and a function $u$ on $M$, such that $\succcurlyeq$ is represented by

$$
S E U: f \mapsto \int_{S} u(f(s)) d P
$$

Theorem 2 (Anscombe and Aumann) The following two statements are equivalent:

(i) Subjective expected utility holds with a nonconstant affine $u$ on $M$. 
(ii) The preference relation $\succcurlyeq$ satisfies the following conditions: (a) nontrivial weak ordering; (b) continuity; (c) monotonicity; (d) independence.

The probabilities $P$ on $S$ are uniquely determined and $u$ on $M$ is unique up to level and unit.

If we apply the above theorem to Example 3, we obtain subjective expected value as in de Finetti (1937; Wakker 2010 Theorem 1.6.1). Thus, two classical derivations of subjective probabilities, by Anscombe and Aumann (1963) and by de Finetti (1937), are based on the same underlying mathematics.

Schmeidler's Choquet Expected Utility. A capacity $v$ on $\mathrm{S}$ maps events to [0, 1], such that $v(\emptyset)=0, v(S)=1$, and $E \supset F \Rightarrow v(E) \geq v(F)$ (set-monotonicity). Unless stated otherwise, we use a rank-ordered notation for acts $f=\left(E_{1}: x_{1}, \cdots, E_{n}: x_{n}\right)$, i.e., $x_{1} \succcurlyeq \cdots \succcurlyeq x_{n}$ is implicitly understood. Let $v$ be a capacity on $S$. Then, for any function $w: S \rightarrow \mathbb{R}$, the Choquet integral of $w$ with respect to $v$, denoted $\int w d v$, is

$$
\int_{0}^{\infty} v(\{s \in S: w(s) \geq \tau\}) d \tau+\int_{-\infty}^{0}[v(\{s \in S: w(s) \geq \tau\})-1] d \tau .
$$

Choquet expected utility holds if there exist a capacity $v$ and a function $u$ on $M$ such that preferences are represented by

$$
C E U: f \mapsto \int_{S} u(f(s)) d v .
$$

Two acts $f$ and $g$ in $\mathcal{A}$ are comonotonic if for no $s$ and $t$ in $S, f(s) \succ f(t)$ and $g(s) \prec g(t)$. Thus, any constant act is comonotonic with any other act. A set of acts is comonotonic if every pair of its elements is comonotonic.

Definition 2 Comonotonic independence holds if

$$
f \succ g \Rightarrow f_{p} c \succ g_{p} c
$$

for all $0<p<1$ and comonotonic acts $f, g$, and $c$.

Under comonotonic independence, preference is not affected by mixing with constant acts (consequences) (with some technical details added in Lemma 3). Because constant acts are comonotonic with each other, comonotonic independence on $\mathcal{A}$ still implies independence on $M$.

Theorem 3 (Schmeidler) The following two statements are equivalent:

(i) Choquet expected utility holds with nonconstant affine $u$ on $M$;

(ii) The preference relation $\succcurlyeq$ satisfies the following conditions: (a) nontrivial weak ordering; (b) continuity; (c) monotonicity; (d) comonotonic independence.

The capacity $v$ on $S$ is uniquely determined and $u$ on $M$ is unique up to level and unit. 
If we apply the above theorem to Example 3, we obtain a derivation of Choquet expected utility with linear utility that is alternative to Chateauneuf (1991, Theorem 1). Cerreia-Vioglio et al. (2015) provide a recent survey of applications.

Comonotonic independence implies a condition assumed by most models for ambiguity proposed in the literature.

Definition 3 Weak certainty independence holds if

$$
f_{q} x \succcurlyeq g_{q} x \Rightarrow f_{q} y \succcurlyeq g_{q} y
$$

for all $0<q<1$, acts $f, g$, and all consequences $x, y$.

That is, preference between two mixtures involving the same constant act $x$ with the same weight $1-q$ is not affected if $x$ is replaced by another constant act $y$. This condition follows from comonotonic independence because both preferences between the mixtures should agree with the unmixed preference between $f$ and $g$ (again, with some technical details added in Lemma 3). Grant and Polak (2013) demonstrated that the condition can be interpreted as constant absolute uncertainty aversion: adding a constant to all utility levels does not affect preference. For a detailed analysis see Skiadas (2013).

\section{Reference dependence in the AA model}

Example 1 violates CEU, as we explain next. In the gain preference $10_{B_{k}} 0 \succ 10_{B_{a}} 0$, the best outcome (= consequence) 10 is preferred under $B_{k}$, implying the strict inequality $v\left(B_{k}\right)>v\left(B_{a}\right)$. In the loss preference $0_{B_{a}}(-10) \succcurlyeq 0_{B_{k}}(-10)$, the best outcome 0 is preferred under $B_{a}$, implying the opposite inequality $v\left(B_{a}\right) \geq v\left(B_{k}\right)$. A contradiction has resulted. This reasoning does not use any assumption about the utilities (10 and -10 in our case) of the outcomes other than that they are of different signs (with $u(0)=0$ ). For later purposes, we show that even weak certainty independence is violated. In the proof of the following observation, we essentially use the linear (probabilistic) mixing of outcomes typical of the AA model.

Observation 1 Example 1 violates comonotonic independence and even weak certainty independence.

Example 1 has confirmed for the AA model what many empirical studies have found for other models: ambiguity attitudes are different for gains than for losses (reviewed by Trautmann and van de Kuilen 2015), violating CEU and most other ambiguity models. Hence, generalizations incorporating reference dependence are warranted. This section presents such a generalization. As in all main results, the analysis will be analogous to Schmeidler's analysis of rank dependence in Choquet expected utility as much as possible. Given this restriction, we stay as close as possible to the analysis of Tversky and Kahneman (1992).

In prospect theory there is a special role for a reference point, denoted $\theta$. In our model it is a consequence that indicates a neutral level of preference. It is often the 
status quo of the decision maker. In Example 1, the deterministic outcome 0 was the reference point. Under the certainty equivalent condition in the AA model, we can always take a deterministic outcome as reference point. Sugden (2003) emphasized the interest of nondegenerate reference points. Many modern studies consider endogenous reference points that can vary (Köszegi and Rabin 2006). Our axiomatization concerns one fixed reference point. Extensions to variable reference points can be obtained by techniques as in Schmidt (2003).

Other consequences are evaluated relative to the reference point. A consequence $f(s)$ is a gain if $f(s) \succ \theta$, a loss if $f(s) \prec \theta$, and it is neutral if $f(s) \sim \theta$. An act $f$ is mixed if there exist $s$ and $t$ in $S$ such that $f(s) \succ \theta$ and $f(t) \prec \theta$. For an act $f$, the gain part $f^{+}$has $f^{+}(s)=f(s)$ if $f(s) \succcurlyeq \theta$ and $f^{+}(s)=\theta$ if $f(s) \prec \theta$. The loss part $f^{-}$is defined similarly, where all gains are now replaced by the reference point. Prospect theory allows different ambiguity attitudes towards gains than towards losses. We therefore use two capacities, $v^{+}$for gains and $v^{-}$for losses. It is more natural to use a dual way of integration for losses. We thus define the dual of $v^{-}$, denoted $\hat{v}^{-}$, by $\hat{v}^{-}(A)=1-v^{-}\left(A^{c}\right)$ for events $A$.

Prospect theory (also called cumulative prospect theory in the literature) holds if there exist two capacities $v^{+}$and $v^{-}$and a function $U$ on consequences with $U(\theta)=$ 0 such that $\succcurlyeq$ is represented by

$$
P T: f \mapsto \int_{S} U\left(f^{+}(s)\right) d v^{+}+\int_{S} U\left(f^{-}(s)\right) d \hat{v}^{-}
$$

We call $U$ in Eq. 7 the (overall) utility function. There is a basic utility $u$ and a loss aversion parameter $\lambda>0$, such that

$$
\begin{array}{r}
U(x)=u(x) \text { if } x>\theta \\
U(x)=u(x)=0 \text { if } x \sim \theta \\
U(x)=\lambda u(x) \text { if } x \prec \theta .
\end{array}
$$

For reasons explained later, we call $\lambda$ the ambiguity-loss aversion parameter (see Section 6). Because $U(\theta)=0$, we now add the scaling convention that also $u(\theta)=0$. For identifying the separation of $U$ into $u$ and $\lambda$, further assumptions are needed. We consider a new kind of separation based on the AA model and the mixture space setup of this paper. Wakker (2010 Chs. 8 and 12) discusses other separations in other models. The parameter $\lambda$ is immaterial for preferences over consequences $M$, affecting neither preferences between gains or losses, nor within. Thus, loss aversion in our model does not affect preferences over $M$ (consequences), that is, over lotteries (risk) in the AA model. It only concerns ambiguity.

For later purposes, we rewrite Eq. 7 as

$$
P T=\sum_{i=1}^{n} \pi_{i} U(f(i))
$$


with decision weights $\pi_{i}$ defined as follows. Assume, for act $\left(E_{1}: x_{1}, \ldots, E_{n}: x_{n}\right)$, the rank-ordering $x_{1} \succcurlyeq \cdots \succcurlyeq x_{k} \succcurlyeq \theta \succcurlyeq x_{k+1} \succcurlyeq \cdots \succcurlyeq x_{n}$. We define

$$
\begin{aligned}
& \text { for } i \leq k: \quad \pi_{i}=\pi_{i}^{+}=v^{+}\left(\cup_{j=1}^{i} E_{j}\right)-v^{+}\left(\cup_{j=1}^{i-1} E_{j}\right) ; \\
& \text { for } i>k: \quad \pi_{i}=\pi_{i}^{-}=v^{-}\left(\cup_{j=i}^{n} E_{j}\right)-v^{-}\left(\cup_{j=i+1}^{n} E_{j}\right) .
\end{aligned}
$$

For gain events, the decision weight depends on cumulative events that yield better consequences. For loss events, the decision weight similarly depends on decumulative events that yield worse consequences. CEU analyzed in the preceding section is the special case of PT where $v^{-}$is the dual of $v^{+}$and $\lambda$ in Eq. 10 is 1.

We next turn to preference conditions that characterize prospect theory. We generalize comonotonicity by adapting a concept of Tversky and Kahneman (1992) to the present context. Two acts $f$ and $g$ are cosigned if they are comonotonic and if there exists no $s$ in $S$ such that $f(s) \succ \theta$ and $g(s) \prec \theta$. Note that, whereas for any act $g$ and any constant act $f, f$ is comonotonic with $g$, an analogous result need not hold for cosignedness. Only if the constant act is neutral, is it cosigned with every other act. This point complicates the proofs in the Appendix. A set of acts is cosigned if every pair is cosigned. We generalize comonotonic independence to allow reference dependence:

Definition 4 Cosigned independence holds if

$$
f \succ g \Rightarrow f_{p} c \succ g_{p} c
$$

for all $0<p<1$ and cosigned acts $f, g$, and $c$.

$\succcurlyeq$ is truly mixed if there exists an act $f$ with $f^{+} \succ \theta$ and $\theta \succ f^{-}$. Double matching holds if, for all acts $f$ and $g, f^{+} \sim g^{+}$and $f^{-} \sim g^{-}$implies $f \sim g$. In a different context, Wakker and Tversky (1993) showed that more general conditions can be used. Our aim here is not to adapt those to the AA model, but we stay as close as possible to Tversky and Kahneman (1992) and use their double matching and true mixedness to achieve maximal comparability and accessibility. We now present the main theorem of this paper.

Theorem 4 Assume true mixedness. The following two statements are equivalent:

(i) Prospect theory holds with $U$ as in Eqs. 8-10.

(ii) The preference relation $\succcurlyeq$ satisfies the following conditions: (a) nontrivial weak ordering; (b) continuity; (c) monotonicity; (d) cosigned independence; (e) double matching.

The capacities are uniquely determined and the global utility function $U$ is unique up to its unit.

Tversky and Kahneman (1992 Theorem 2) provided a behavioral foundation of prospect theory in a Savagean-like framework, where outcomes are monetary with 
no probabilities or multiple stages involved. They thus avoided the ancillary assumptions of the AA model. As a price to pay, they did not have the convenient mixture structure typical of the AA model, making measurements and analyses of behavioral properties more difficult. They used conditions similar to (a)-(c) that are standard in most behavioral foundations, and also condition (e). Their main axiom, signcomonotonic tradeoff consistency, had to be more complex than our main axiom (d). Several generalizations were provided for the Savagean framework, mainly weakening true mixedness and double matching, with extensions to multiattribute outcomes, connected topological outcome spaces, and nonsimple prospects, but always using a complex sign-comonotonic tradeoff consistency (Bleichrodt and Miyamoto 2003; Bleichrodt et al. 2009; Köbberling and Wakker 2003; Kothiyal et al. 2011; Wakker 2010 Theorem 12.3.5; Wakker and Tversky 1993). Closest to our theorem is Schmidt and Zank's (2009) result, who used linear utility with respect to monetary outcomes, as in Example 3. Our paper provides the first axiomatization of PT for the AA model. The difference between the aforementioned results and ours is similar to that between Savage (1954)/Wakker (2010 Theorem 4.6.4) versus Anscombe and Aumann (1963), or Gilboa (1987)/Wakker (1989) versus Schmeidler (1989).

We give the proof of the following observation in the main text because it is clarifying.

Observation 2 Example 1 can be accommodated by prospect theory.

Proof To see that the observation holds, choose, in Example $1, v^{+}\left(B_{k}\right)>v^{+}\left(B_{a}\right)$, $v^{+}\left(R_{k}\right)>v^{+}\left(R_{a}\right), v^{-}\left(B_{k}\right)>v^{-}\left(B_{a}\right)$, and $v^{-}\left(R_{k}\right)>v^{-}\left(R_{a}\right)$. Remember here that large values of $v^{-}$correspond with low values of its dual capacity as used in the Choquet integral.

We can take $v^{-}$different than $v^{+}$, letting $v^{-}$accommodate ambiguity seeking in agreement with empirical evidence.

Observation 3 For the preference relation $\succcurlyeq$ restricted to consequences, there exists an affine representation $u$ if and only if $\succcurlyeq$ satisfies nontrivial weak ordering, continuity, and cosigned independence.

For consequences, cosigned independence means that independence in Definition 1 is restricted to cases where the consequences $x, c, y$ are all better or all worse than the reference point.

\section{Measurements and interpretations of ambiguity loss aversion}

This section considers a number of interpretations of the ambiguity-loss aversion parameter $\lambda$ in Theorem 4 and Eqs. 8-10. We first show how $\lambda$ can be directly revealed from preference. This direct measurement is typical of the AA model with its mixture operation, and cannot be used in other models. 
Observation 4 For all $f$ in $\mathcal{A}, x^{+}, x^{-} \in M$, and $\lambda \in \mathbb{R}$, if $f \sim \theta, f^{+} \sim x^{+} \succ \theta$, and $f^{-} \sim x^{-} \prec \theta$, then $x_{\frac{1}{1+\lambda}}^{+} x^{-} \sim \theta$.

In other words, with $f, x^{+}$, and $x^{-}$as in the observation, we find $p$ such that $x_{p}^{+} x^{-} \sim \theta$, and then solve $\lambda$ from $\frac{1}{1+\lambda}=p\left(\lambda=\frac{1-p}{p}\right)$. The condition in the theorem is intuitive: The indifference $x_{\frac{1}{1+\lambda}}^{+} x^{-} \sim \theta$ shows that, when mixing consequences (lotteries in the AA model), the loss must be weighted $\lambda$ times more than the gain to obtain neutrality. Under ambiguity, however, $f$ combines the preference values of $x^{+}$and $x^{-}$in an "unweighted" manner (see the unweighted sum of the gain- and loss-part in Eq. 7), leading to the same neutrality level. Apparently, under ambiguity, losses are weighted $\lambda$ times more than when mixing consequences (risk in the AA model). In the AA model, with consequences referring to lotteries and decision under risk, $\lambda$ indicates how much more losses are overweighted under ambiguity than they are under risk. Thus, $\lambda$ purely reflects ambiguity attitude.

In the smooth ambiguity model (Klibanoff et al. 2005), ambiguity attitudes depend entirely on the outcomes faced (in the domain of its second-order ambiguity-utility transformation function $\varphi$ ), and sign dependence is a special case of such a dependency. The smooth model can accommodate extra loss aversion due to ambiguity in the same way as our parameter $\lambda$ does: through a kink of its $\varphi$ at 0 . The smooth model differs from our model because we capture other aspects of ambiguity attitudes through functions operating on events, rather than on outcomes.

For a first prediction on values of $\lambda$, we consider an extreme view on loss aversion for the AA model. It entails that all loss aversion shows up under risk, and that no additional loss aversion is expected due to ambiguity. This interpretation is most natural if loss aversion only reflects extra suffering experienced under losses, rather than an overweighting of losses without them bringing disproportional suffering when experienced. That is, this extreme interpretation ascribes loss aversion entirely to the (utility of) consequences. Then it is natural to predict that $\lambda=1$, with no special role for ambiguity. We display the preference condition axiomatizating this prediction and showing how the prediction can be tested:

Neutral ambiguity-loss aversion holds if $\lambda=1$ in Observation 4.

A less extreme interpretation of ambiguity-loss aversion is as follows: There is loss aversion under risk, which can be measured in whatever is the best way provided in the literature. ${ }^{12}$ For monetary outcomes with a fixed reference point as considered in this paper, loss aversion will generate a kink of risky utility at that reference point. As an aside, in our model loss aversion under risk does not imply violations of expected utility and is fully compatible with our AA model, simply giving a kinked function $u$. Ambiguity can give extra loss aversion and it can amplify $(\lambda>1)$ or moderate $(\lambda<1)$ it. The following preference condition characterizes $\lambda$ :

\footnotetext{
${ }^{12}$ Many studies have discussed ways to measure loss aversion under risk (Abdellaoui et al. 2007). This debate is outside the scope of this paper.
} 
Nonneutral ambiguity-loss aversion. For all $f$ in $\mathcal{A}, x^{+}, x^{-} \in M$, and $\lambda \in \mathbb{R}$, if $f \sim \theta, f^{+} \sim x^{+} \succ \theta$, and $f^{-} \sim x^{-} \prec \theta$, then $x_{0.5}^{+} x^{-} \succ \theta$ if and only if $\lambda>1$, and $x_{0.5}^{+} x^{-} \prec \theta$ if and only if $\lambda<1$.

Abdellaoui et al. (2016) measured loss aversion under risk and ambiguity separately and found them to be the same. Baltussen et al. (2016) also found them to be the same in one treatment (outside the "limelight"), but not in the other (in the limelight).

In the two-stage AA model, some consequences are outcomes and others are lotteries. Reference dependence in this paper takes lotteries as a whole, and their indifference class determines if they are gains or losses. This is analogous to the way in which Schmeidler (1989) modeled rank dependence, which also concerned lotteries as a whole. Another approach can be considered, both for reference dependence and rank dependence, where outcomes within a lottery are perceived as gains or losses and are weighted in a rank dependent manner. Here, as elsewhere, we followed Schmeidler's approach. Tversky and Kahneman (1981, p. 456 penultimate paragraph) recommended this approach for reference dependence. In the rAA model, subjects are never required to perceive whole lotteries in a reference or rank dependent manner, but we implement it ourselves, and subjects only see the CAs that we inserted. Hence, the above issue is no problem for us.

\section{Discussion}

Kreps (1988 p. 101) wrote about the non-descriptive nature of two-stage acts in the AA model:

imaginary objects. ... makes perfectly good sense in normative applications ... But this is a very dicey and perhaps completely useless procedure in descriptive applications. ... what sense does it make ... because the items concerned don't exist? I think we have to view the theory to follow [the traditional twostage AA model] as being as close to purely normative as anything that we do in this book.

A pragmatic objection can be raised against the rAA model. The mixture operation of outcomes is not as easy to implement as in the original AA model. Now a mixture is not done by just multiplying probabilities, but it requires observing an indifference. But such observations are easy to obtain, as our experiment demonstrated. They concern stimuli that are easier to understand for subjects than two-stage acts.

We next analyze to what extent we have succeeded in avoiding violations of EU in the rAA model. Because we always assign a non-negligible probability $(0.2$ in our experiment) to the best outcome and to the worst outcome, for the preferences that we consider, the nonlinear processing of probability typical of nonEU is only relevant in the middle of the domain, bounded away from $p=0$ and $p=1$. The common empirical finding is that deviations from linearity mostly occur at the boundaries (Baucells and Villasis 2015; Starmer 2000; Tversky and Kahneman 1992; Viscusi 
and Evans 2006; Wakker 2010 p. 208). ${ }^{13}$ Hence, the deviations from EU are weak for the stimuli in the rAA model. We recall here that loss aversion is incorporated in $u$, as a kink at zero.

Some papers considered relaxations of the four assumptions of the AA model listed in Section 1. Dean and Ortoleva (2017 Footnote 7) suggested using the rAA domain, but did not elaborate on it and still used the second ancillary assumption of AA (backward induction). They did however relax the first ancillary assumption of EU. Their axioms used an endogenous utility midpoint operation, which serves a purpose similar to our substitution of $C A_{i} \mathrm{~s}$ in Fig. 4. They are, to our best knowledge, the first who succeeded in using the AA model without assuming EU in the second stage. Borah and Kops (2016) analyzed the AA model theoretically on a restricted domain similar to ours. In a theoretical study, Bommier (2017) did consider two-stage AA acts, but he neither assumed EU for risk nor backward induction, instead using a sort of dual forward-induction type optimization. He analyzed ambiguity aversion as defined in his setting, but did not consider reference dependence.

\section{Conclusion}

To date, the AA ambiguity model could only be used for normative purposes (Kreps 1988 p. 101). We have made it suitable for descriptive purposes. We demonstrated how the two major descriptive problems (violations of EU for risk and of backward induction) can be resolved through a reduced AA model (rAA). The rAA model introduces an imaginary AA twin $\succcurlyeq^{*}$ for a real decision maker $\succcurlyeq$, where every $\succcurlyeq^{*}$ relationship can be derived from an rAA $\succcurlyeq$ relationship through Fig. 3. Next, we can apply any AA theorem available in the literature to $\succcurlyeq^{*}$, and its conclusions regarding ambiguity attitudes are valid for the real decision maker $\succcurlyeq$. In a simple experiment we showed how the rAA model can be implemented and how the AA model can be tested in general. A formal model-theoretic isomorphism showed that the rAA model maintains the full analytical power of the AA model.

We conducted the first empirical test of a preference condition in the AA model that is not confounded by violations of the ancillary assumptions. This test sufficed to falsify two assumptions of the majority of AA ambiguity theories today: weak certainty independence and reference independence - the latter often assumed implicitly. We benefited from an additional advantage of the reduced AA model: it only needs one-stage stimuli and those are easy to understand for subjects.

To accommodate the violations found, we introduced a reference dependent generalization of the first decision model of ambiguity that received a behavioral foundation: Schmeidler's (1989) Choquet expected utility. Our generalization amounts to extending the AA model to prospect theory. We provided a behavioral foundation. Topics for future research include the development of reference dependent generalizations of the many other ambiguity theories in the literature, and empirical tests of such models. We hope that our paper will advance descriptive applications of ambiguity AA theories, having removed the major obstacles.

\footnotetext{
${ }^{13}$ As a technical point, if probability weighting is more (or less) steep in the interior for losses than for gains, this can be captured by ambiguity-loss aversion.
} 
Open Access This article is distributed under the terms of the Creative Commons Attribution 4.0 International License (http://creativecommons.org/licenses/by/4.0/), which permits unrestricted use, distribution, and reproduction in any medium, provided you give appropriate credit to the original author(s) and the source, provide a link to the Creative Commons license, and indicate if changes were made.

\section{Appendices: Proofs and an isomorphism}

\section{Appendix A: Preparation}

Several results in the ambiguity literature (e.g., Schmeidler 1989), were formulated for the two-stage AA model, and not for general mixture spaces as we use them. These results can routinely be transferred to acts for general mixture spaces. For example, this can be inferred by verifying that all those proofs remain valid for general mixture spaces, as do the proofs provided by Ryan (2009). Another way to see this point is as follows.

In all our results, Theorem 1 (or Observation 3) gives an affine representation $u$ on $M$. We replace all consequences by their $u$ values (effectively, collapsing indifference classes of consequences), endowing those with the natural mixture on real numbers. By monotonicity, we thus collapse indifference classes of acts. The newly constructed space is a two-stage AA model, with the utility function on consequences being the identity function. All preference conditions defined in this paper are preserved under the transformation used. Hence, we can use the existing theorems in the literature. They give the corresponding theorems on the underlying general mixture space. We have thus shown:

Observation 5 All cited preference foundations for AA theories hold for general mixture spaces.

\section{Appendix B: Proof of Observation 3: cosigned expected utility}

A nonloss is a consequence that is a gain or is neutral, and a nongain is a consequence that is a loss or is neutral. We first derive a preparatory lemma.

Lemma 1 Assume that the preference relation $\succcurlyeq$, restricted to consequences, satisfies weak ordering, continuity, and cosigned independence. If $x$ and y are nonlosses, then so are all $x_{p} y$ for $0 \leq p \leq 1$. If $x$ and $y$ are nongains, then so are all $x_{p} y$ for $0 \leq p \leq 1$.

Proof Assume the conditions in the lemma. We consider the case of nonlosses $x, y$. Assume, for contradiction, $x_{q} y \prec \theta$ for some $q$. Continuity readily implies existence of a largest $p<q$ such that $x_{p} y \sim \theta$ and a smallest $r>q$ such $x_{r} y \sim \theta$. Define $x^{\prime}=$ $x_{p} y$ and $y^{\prime}=x_{r} y$. Then $x^{\prime}$ and $y^{\prime}$ are neutral but, by continuity, every $x_{p^{\prime}}^{\prime} y^{\prime}$ must be a loss. The set of $x_{p^{\prime}}^{\prime} y^{\prime}\left(0 \leq p^{\prime} \leq 1\right)$ is cosigned, implying that von NeumannMorgenstern independence holds here without a cosignedness restriction. $x^{\prime} \succ x_{1 / 3}^{\prime} y^{\prime}$ and independence imply that their $0.5-0.5$ mixture is strictly preferred to $x_{1 / 3}^{\prime} y^{\prime}$ 
(take $c=x_{1 / 3}^{\prime} y^{\prime}$ in the definition of cosigned independence), implying that $x_{2 / 3}^{\prime} y^{\prime} \succ$ $x_{1 / 3}^{\prime} y^{\prime}$. In contradiction with this, $y^{\prime} \succ x_{2 / 3}^{\prime} y^{\prime}$ and independence imply that their $0.5-$ 0.5 mixture is strictly preferred to $x_{2 / 3}^{\prime} y^{\prime}$, implying $x_{1 / 3}^{\prime} y^{\prime} \succ x_{2 / 3}^{\prime} y^{\prime}$. A contradiction has resulted.

We now turn to the proof of Observation 3. Necessity of the preference conditions is obvious. We hence assume these preference conditions and derive an affine representation. We assume the $v N M$ axioms (the axioms in Theorem 1) for $\succcurlyeq$ over consequences with, however, independence weakened to sign-independence: $x \succ y \Rightarrow x_{p} z \succ y_{p} z$ only if either all consequences are nonlosses or they all are nongains. By true mixedness, there exist consequences $\alpha$ and $\beta$ with $\alpha \succ \theta \succ \beta$, and we will use these consequences in the following derivation.

Lemma 1 implies that the set of nonlosses is a mixture set (closed under mixing). On this set, all vNM axioms are satisfied, and an affine representing functional $u^{+}$ is obtained. We normalize $u^{+}(\theta)=0, u^{+}(\alpha)=1$. We similarly obtain an affine $u^{-}$ on nongains. To extend the representation and its affinity to mixed consequences, we define an as-if gain preference relation $\succcurlyeq^{+}$over consequences, including losses, as follows. It agrees with $\succcurlyeq$ for gains, as we will see, and affinity extends it to losses: $x \succcurlyeq^{+} y$ if there exists $p<1$ such that $\alpha_{p} x \succcurlyeq \alpha_{p} y \succcurlyeq \theta$. We first show that the choice of $p$ in the definition of $\succcurlyeq^{+}$is immaterial.

Lemma 2 If $x \succcurlyeq^{+} y$ then $\alpha_{p} x \succcurlyeq \alpha_{p} y$ for all $p>0$ for which both mixtures are nonlosses.

Proof Consider $\alpha_{p} x, \alpha_{p} y, \alpha_{r} x$, and $\alpha_{r} y$, and assume that all are nonlosses. Assume $p>r$. Then $\alpha_{p} x$ is a mixture of $\alpha_{r} x$ and $\alpha$, and $\alpha_{p} y$ is a mixture of $\alpha_{r} y$ and $\alpha$, where both mixtures use the same weights $((1-p) /(1-r)$ and $(p-r) /(1-r))$. By the affine representation for nonlosses, the preference between $\alpha_{p} x$ and $\alpha_{p} y$ is the same as between $\alpha_{r} x$ and $\alpha_{r} y$.

The above lemma shows that $\succcurlyeq^{+}$indeed agrees with $\succcurlyeq$ for nonlosses (take $p=0$ ). To see that it establishes an affine extension for losses, we briefly show that $\succcurlyeq^{+}$ satisfies all usual vNM axioms, also on losses. Completeness, transitivity, nontriviality, and independence all readily follow from the definition of $\succcurlyeq^{+}$by taking a mixture weight $p$ in its definition so close to 1 that this same mixture weight $p$ can be used for all consequences concerned in the axioms. This also holds for continuity, where, applying it to $\succcurlyeq$ and $\alpha_{p} f, \alpha_{p} g$, and $\alpha_{p} h$ with $p$ sufficiently close to 1 , implies it for $\succcurlyeq^{+}, f, g$, and $h$. All vNM axioms are satisfied for $\succcurlyeq^{+}$, giving an affine representation, denoted $u^{+}$of $\succcurlyeq+$ and, hence, also of $\succcurlyeq$ on all nonlosses.

We similarly define an as-if loss preference relation: $x \succcurlyeq^{-} y$ if there exists $p<1$ such that $\theta \succcurlyeq \beta_{p} x \succcurlyeq \beta_{p} y$. We similarly obtain an affine representation, denoted $u^{-}$, of $\succcurlyeq^{-}$that agrees with $\succcurlyeq$ for all nongains. $u^{+}$and $u^{-}$both represent $\succcurlyeq$ on the set of neutral consequences. We show that this overlap is big enough to ensure that the two representations are identical.

We can set $u^{+}(\theta)=0=u^{-}(\theta)$. By continuity, we can take $0<p<1$ such that $\alpha_{p} \beta \sim \theta$. Because $u^{-}$represents $\succcurlyeq$ for losses, $u^{-}(\beta)<u^{-}(\theta)=0$, and 
hence $u^{-}(\alpha)>0$. We normalize $u^{-}(\alpha)=u^{+}(\alpha)=1$. Indifferences $\alpha_{q} \gamma \sim \theta$ for losses $\gamma$, and the affine representations, imply that $u^{+}=u^{-}$for losses $\gamma$. Thus, $u^{+}(\beta)=u^{-}(\beta)$. This and indifferences $\delta_{r} \beta \sim \theta$ imply that $u^{+}=u^{-}$for gains $\delta$ too. Hence, $u^{+}=u^{-}$everywhere, and $u^{+}=u^{-}$. Consequently, both these functions represent $\succcurlyeq$ on nonlosses and on nongains. They also represent preferences between gains and losses properly, assigning positive values to the former and negative values to the latter. We have thus obtained an affine representation $u^{+}=u^{-}$of $\succcurlyeq$, implying all the vNM conditions for consequences without sign restrictions. We denote $u=$ $u^{+}=u^{-}$. This completes the proof of Observation 3 .

\section{Appendix C: Proof of Theorem 4}

We first show that the implications in the definitions of independence can be reversed. We use the term strong (comonotonic/cosigned) independence to refer to these reinforced versions.

Lemma 3 Assume that $\succcurlyeq$ is a continuous weak order. Then the reversed implications in Definitions 1, 2, and 4 also hold.

Proof Assume the conditions in the lemma and the implication of the definition considered. Consider three acts $f, g, h$. If $f, g, h$ are comonotonic (or cosigned), then so is the mixture set of all their mixtures, by Observation 3. In each case, independence therefore holds on the mixture set considered without a comonotonicity/cosignedness restriction, and we have the usual axioms that imply expected utility and the reversed implications of Lemma 3.

NeCessity of the Preference Conditions in Theorem 4; i.e., (i) implies (ii): We assume (i), PT, and briefly indicate how cosigned independence is implied. The other conditions are routine. Consider cosigned $f, g, c$. We may assume a common partition $E_{1}, \ldots, E_{n}$ such that the consequences of the acts depend on these events. Because of cosignedness we can have

$$
h_{1} \succcurlyeq \cdots \succcurlyeq h_{k} \succcurlyeq \theta \succcurlyeq h_{k+1} \succcurlyeq \cdots \succcurlyeq h_{n}
$$

for all $h$ equal to $f, g$, or $c$, or a mixture of these acts. For example, if for $i$ there exists a $h^{\prime}$ from $\{f, g, c\}$ with $h_{i}^{\prime}$ a gain, then all $h_{i}$ s are nonlosses and $i \leq k$. If $h_{j} \succ h_{i}$ for a $h^{\prime}$ from $\{f, g, c\}$, then $h_{j} \succcurlyeq h_{i}$ for all three acts, and $j<i$. Thus, we can use the same decision weights (Eqs. 12 and 13) for all three acts and for all their mixtures. It implies that $P T\left(f_{p} c\right)=p P T(f)+(1-p) P T(c)$, with the same equality for $g$ instead of $f$. This implies cosigned independence.

Sufficiency of the Preference Conditions in Theorem 4 ((ii) implies (i)). In Observation 3 we derived expected utility for consequences if only cosigned independence is assumed. In agreement with the definition of prospect theory, we normalize expected utility for consequence $\theta$ such that $u(\theta)=0$ and for some consequence (existing because of true mixedness) $\check{\alpha} \succ \theta$ such that $u(\check{\alpha})=1$. Let a nonloss act be an act $g$ such that $g(s)$ is a nonloss for every $s$. A nongain act is 
defined similarly. By Lemma 1, the set of nonloss acts is closed under mixing, and so is the set of nongain acts. By Schmeidler's Theorem 3, there exists a CEU functional $C E U^{+}=\int_{S} u\left(g^{+}(s)\right) d v^{+}$on the nonloss acts $g^{+}$that represents $\succcurlyeq$ there $\mathrm{CEU}^{-}$is similar.

By true mixedness, there exists a truly mixed act. By monotonicity, we can replace all nonloss consequences of the act by its maximal consequence, and all loss consequences by its minimal consequence, without affecting its true mixedness. The act now only has two consequences and can be written as $\gamma_{F} \beta$ with $\gamma>\theta>\beta$. ( $\gamma$ abbreviates good (or gain) and $\beta$ abbreviates bad.) By continuity, we assume that $\gamma_{F} \beta \sim \theta$, by either improving (by mixing with $\theta$ ) $\beta$ or worsening (by mixing with $\theta$ ) $\gamma \cdot \gamma_{F} \beta$ will be used for calibrating the $P T$ functional, and is called the calibration act.

We now define a functional $P T^{+}$on nonloss acts and a functional $P T^{-}$on nongain acts, and a prospect theory functional $P T$ that is the sum of those two. Next we show that $P T$ represents preference. More precisely, we define

$$
P T(f)=P T^{+}\left(f^{+}\right)+P T^{-}\left(f^{-}\right)=C E U^{+}\left(f^{+}\right)+\lambda C E U^{-}\left(f^{-}\right),
$$

where $\lambda>0$ is such that $P T\left(\gamma_{F} \beta\right)=0$. Thus, $P T\left(\gamma_{F} \beta\right)=P T^{+}\left(\gamma_{F} \theta\right)+$ $P T^{-}\left(\theta_{F} \beta\right)$, and $\lambda=-C E U^{+}\left(\gamma_{F} \theta\right) / C E U^{-}\left(\theta_{F} \beta\right)$. We define $c$ as the $P T$ value of the gain part of $\gamma_{F} \beta$; i.e.,

$$
c=P T^{+}\left(\gamma_{F} \theta\right)>0 .
$$

This $c$ is minus the $P T$ value of the loss part of $\gamma_{F} \beta$; i.e., $P T^{-}\left(\theta_{F} \beta\right)=-c$.

$P T$ represents preference on all nonloss acts, and also on all nongain acts. Because it also compares nonloss acts properly with nongain acts (this holds for every $\lambda>0$ ), it is representing on the union of these, which is the set of all nonmixed acts. We call an act $f$ proper if $P T(f)=P T(g)$ for some nonmixed act $g$ with $f \sim g$. To prove that $P T$ is representing, it suffices, by transitivity, to show that all acts are proper, and this is what we will do. That is, we use the nonmixed acts for calibrating $P T$ relative to preferences. We start with a set of binary acts cosigned with the calibration act: $\mathcal{A}_{F}$ is defined as the set of all acts $\delta_{F} \alpha$ with $\delta \succcurlyeq \theta \succcurlyeq \alpha$.

\section{Lemma 4 All acts in $\mathcal{A}_{F}$ are proper.}

Proof In this proof we only consider acts from $\mathcal{A}_{F}$. All these acts are cosigned, implying that we can use cosigned independence for all mixtures. We choose particular nonmixed acts. For any act $f$ we find a nonmixed equivalent $g$ defined as follows. Let $x$ be a consequence such that with $g=x_{F} \theta$ we have $P T(g)=P T(f)$. By continuity of $P T$, such an $x$ always exists. Thus, $g$ is a nonmixed binary act with the same $P T$ value as $f$, but it is in $\mathcal{A}_{F}$ and is cosigned with $f$ and $\theta$. We will demonstrate properness on $A_{F}$ by showing that each act is equivalent to a nonmixed equivalent.

CASE 1 [acts with $P T$ value zero]: Let $P T(f)=0$. Define $a=P T^{+}\left(f^{+}\right)=$ $-P T^{-}\left(f^{-}\right) \geq 0 . \theta$ is a nonmixed equivalent of $f$. We show that $f \sim$ $\theta$.

CASE 1.1: $a \leq c$ ( $c$ as in Eq. 16). $P T^{+}\left(f^{+}\right)=\frac{a}{c} P T^{+}\left(\gamma_{F} \theta\right)$. By CEU for nonlosses, $f^{+} \sim\left(\gamma_{F} \theta\right)_{a / c} \theta$. Similarly, $f^{-} \sim\left(\theta_{F} \beta\right)_{a / c} \theta$. By double 
matching, $f \sim\left(\gamma_{a / c} \theta\right)_{F}\left(\beta_{a / c} \theta\right)=\left(\gamma_{F} \beta\right)_{a / c} \theta \sim \theta$ (the last indifference by cosigned independence). By transitivity, $f \sim \theta$ and $f$ is proper.

CASE 1.2: $a>c$. We consider a mix of $f$ with $\theta, f_{p} \theta$. From the definition of the $P T$ functional we have $P T\left(f_{p} \theta\right)=p P T(f)=0$ and $\left.P T^{+}\left(f_{p} \theta\right)^{+}\right)=-P T^{-}\left(\left(f_{p} \theta\right)^{-}\right)=p a$. We choose $p$ so small that $0<p a<c$. From Case 1.1 we have $f_{p} \theta \sim \theta$. By strong cosigned independence, this implies $f \sim \theta . f$ is proper.

CASE 2 [acts with positive $P T$ value]: Let $P T(f)>0$. By continuity and the definition of $P T$, there exists a consequence $\delta$ between $\theta$ and the maximal consequence in $f$ such that $P T\left(\delta_{F} \theta\right)=P T(f)>0$. $\delta_{F} \theta$ is a nonmixed equivalent of $f$. Define $a^{+}=P T^{+}\left(f^{+}\right)$and $a^{-}=-P T^{-}\left(f^{-}\right)$. Then $P T\left(\delta_{F} \theta\right)=a^{+}-a^{-}$.

CASE 2.1: $a^{+} \leq c$ (hence $a^{-}<c$ ). Write $b^{+}=a^{+} / c$ and $b^{-}=a^{-} / c$.

$P T^{+}\left(f^{+}\right)=b^{+} P T^{+}\left(\gamma_{F} \theta\right)$ and $P T^{-}\left(f^{-}\right)=b^{-} P T^{-}\left(\theta_{F} \beta\right)$. Then it follows from CEU for gains that $f^{+} \sim\left(\gamma_{F} \theta\right)_{b^{+}} \theta$. For the loss part of $f$, we similarly have $f^{-} \sim\left(\theta_{F} \beta\right)_{b^{-}} \theta$. By double matching, $f \sim\left(\gamma_{b^{+}} \theta\right)_{F}\left(\beta_{b^{-}} \theta\right)$. We now isolate a symmetric component with absolute prospect theory value $a^{-}$for the gain part and the loss part (this was the step most difficult to find in this paper): $\left(\gamma_{b^{+}} \theta\right)_{F}\left(\beta_{b^{-}} \theta\right)=$ $\left(\gamma_{F} \beta\right)_{b^{-}}\left[\left(\gamma_{\frac{b^{+}-b^{-}}{1-b^{-}}} \theta\right)_{F} \theta\right] \sim(\theta)_{b^{-}}\left[\left(\gamma_{\frac{b^{+}-b^{-}}{1-b^{-}}} \theta\right)_{F} \theta\right]$ $\left(\gamma_{\left(b^{+-} b^{-}\right)} \theta\right)_{F} \theta=f^{*}$. From $P T\left(f^{*}\right)=c\left(b^{+}-b^{-}\right)=a^{+}-a^{-}=$ $P T\left(\delta_{F} \theta\right)$ and CEU for nonlosses it follows that $f^{*} \sim \delta_{F} \theta$. By transitivity, $f \sim \delta_{F} \theta$. $f$ is proper.

CASE 2.2: $a^{+}>c$. We mix $f$ and $\delta_{F} \theta$ with $\theta$ to obtain $f_{\downarrow}=f_{p} \theta$ and $\left(\delta_{F} \theta\right)_{\downarrow}=$ $\left(\delta_{F} \theta\right)_{p} \theta$. We define $a_{\downarrow}^{+}=P T\left(\left(f_{p} \theta\right)^{+}\right)$, which is $p a^{+}$, and $a_{\downarrow}^{-}=$ $P T\left(\left(f_{p} \theta\right)^{-}\right)$, which is $p a^{-}$. We choose $p$ so small that $a_{\downarrow}^{-}<a_{\downarrow}^{+}<$ $c$. From prospect theory we have $P T\left(f_{\downarrow}\right)=P T\left(\left(\delta_{F} \theta\right)_{\downarrow}\right)$, which, by Case 2.1, implies $f_{\downarrow} \sim\left(\delta_{F} \theta\right)_{\downarrow}$. Because $f, \delta_{F} \theta$, and $\theta$ are cosigned, $f_{p} \theta \sim\left(\delta_{F} \theta\right)_{p} \theta$ implies $f \sim \delta_{F} \theta$. Again, $f$ is proper.

CASE 3 [Acts with negative $P T$ value]: Let $P T(f)<0$. This case is similar to Case 2.

We have demonstrated that all acts in $\mathcal{A}_{F}$ are proper.

We next show that all acts are proper. Consider a general act $g$, and event $E$ such that $g$ yields nonlosses on $E$ and losses on $E^{c}$.

CASE 1: There exists a matching act $f \in \mathcal{A}_{F}$ such that $P T^{+}\left(f^{+}\right)=P T^{+}\left(g^{+}\right)$ and $P T^{-}\left(f^{-}\right)=P T^{-}\left(g^{-}\right)$. Hence, $P T(f)=P T(g)$, and from CEU for nonlosses and for nongains we have $f^{+} \sim g^{+}$and $f^{-} \sim g^{-}$. From double matching, $f \sim g$. Because $f$ and $g$ have the same $P T$ value and are equivalent, and $f$ is proper, it follows that $g$ is also proper.

CASE 2. There exists no matching act $f \in \mathcal{A}_{F}$ for $g$ as in Case 1 . We mix act $g$ with $\theta$ to obtain an act $g_{\downarrow}=g_{p} \theta$. We choose $p$ so small that we find a matching act $f_{\downarrow} \in A_{F}$, i.e., $P T^{+}\left(f_{\downarrow}^{+}\right)=P T^{+}\left(g_{\downarrow}^{+}\right)$and $P T^{-}\left(f_{\downarrow}^{-}\right)=P T^{-}\left(g_{\downarrow}^{-}\right)$. Thus, $P T\left(f_{\downarrow}\right)=P T\left(g_{\downarrow}\right)$, and Case 1 implies $f_{\downarrow} \sim g_{\downarrow}$. 
Let $\tilde{g}=x_{E} \theta$ be the nonmixed equivalent of $g$. Let $\tilde{g_{\downarrow}}=x_{E}^{\prime} \theta$ similarly be the nonmixed equivalent of $g_{\downarrow}$. We have $P T\left(g_{\downarrow}\right)=P T\left(\tilde{g}_{\downarrow}\right)$, and because of Case 1 this implies $\tilde{g}_{\downarrow} \sim g_{\downarrow}$. Because $g, \tilde{g}$, and $\theta$ are cosigned, $g_{p} \theta \sim \tilde{g}_{p} \theta$ implies $g \sim \tilde{g}$. Thus, $g$ is proper. We have proved sufficiency of the preference conditions.

UniQueness Results Uniqueness of $v^{+}\left(v^{-}\right)$follows from Schmeidler's Theorem 3 applied to nonloss (nongain) acts. It is obvious that the unit of utility can be multiplied by any positive constant. We show that no other change is possible. Restricting attention to nonloss consequences shows, by Schmeidler's theorem, that $u$, when restricted to nonlosses, is unique up to a unit, given that the scale $u(\theta)=0$ is fixed. Similarly, restricting attention to nongain consequences shows that $u$, when restricted to nongains, is unique up to a unit that, a priori, might be different than for gains. However, the equivalence $\gamma_{F} \beta \sim \theta$ shows that the unit of losses is joined with that of gains, and a change of one implies the same change of the other. Hence, only one unit of utility is free to choose.

\section{Appendix D: Remaining proofs}

Proof of Observation $1\left(20_{B_{k}} 0\right)_{\frac{1}{2}}(0) \succ\left(20_{B_{a}} 0\right)_{\frac{1}{2}}(0)$ and $\left(20_{B_{k}} 0\right)_{\frac{1}{2}}(-20) \preccurlyeq$ $\left(20_{B_{a}} 0\right)_{\frac{1}{2}}(-20)$ violate weak certainty independence, but are exactly the preferences in Example 1 under the AA model. To see the latter point, the left mixture in the first preference, for example, yields $20_{\frac{1}{2}} 0=10$ under event $B_{k}$.

Proof of Observation $4 P T(f)=C E U^{+}\left(f^{+}\right)+\lambda C E U^{-}\left(f^{-}\right)=u\left(x^{+}\right)+$ $\lambda u\left(x^{-}\right)=P T(\theta)=0$ implies $u\left(x^{+}\right)=-\lambda u\left(x^{-}\right)$. Then for $g=x_{p}^{+} x^{-} \sim \theta$, $P T(g)=p u\left(x^{+}\right)+(1-p) u\left(x^{-}\right)=p(-\lambda) u\left(x^{-}\right)+(1-p) u\left(x^{-}\right)=0$, implying $-\lambda p+1-p=0 \Leftrightarrow p=\frac{1}{1+\lambda}$.

\section{Appendix E: An isomorphism between our reduced and the full AA model}

This appendix formally shows that the procedure described in Section 2, considering only a subdomain of the preferences (formalized through an rAA model) as used in the experiment, is model-theoretically isomorphic to the full AA model. The rAA model thus contains all information of the complete model, and we can indeed use all techniques of the full AA model despite our restricted domain. We first present the usual AA model formally. We assume Example 2. Generic notation: $\alpha, \beta, x_{i}, y_{i}$ for outcomes; $x=\left(p_{1}: x_{1}, \ldots, p_{m}: x_{m}\right)$, with the obvious interpretation, for lotteries. General acts are also called (two-stage) acts because there are two stages of uncertainty.

Preferences over lotteries induce preferences over outcomes through degenerate lotteries. A certainty equivalent $(C E)$ of a lottery is an outcome that is equivalent to that lottery. Under EU, it agrees with the CA of the lottery. The certainty equivalent condition means that there exists a unique certainty equivalent for each lottery. Uniqueness can always be achieved by collapsing indifference classes of outcomes. 
A function $u$ on $L$ is expected utility $(E U)$ if $u\left(\left(p_{1}: x_{1}, \ldots, p_{m}: x_{m}\right)\right)=\sum_{i=1}^{m} p_{i} u\left(x_{i}\right)$ and it represents $\succcurlyeq$ on $L$. This is equivalent to affinity of $u$. We use the same symbol $u$ for the function defined on $X$ and its expectation defined on $L$. We sometimes call $u$ on $L$ the risky utility function. We defined the (two-stage) AA model in the main text by EU maximization over lotteries (w.r.t. $u$ ) plus backward induction, which is equivalent to monotonicity under the following richness, which is assumed in this appendix:

Assumption 1 A nontrivial weak order $\succcurlyeq$ is given on the set $\mathcal{A}$ of acts, with a best outcome $B$ and a worst outcome $W$. The CE condition is satisfied.

The best and worst outcomes are defined by $B \succcurlyeq \alpha \succcurlyeq W$ for all outcomes $\alpha$. They simplify utility scalings and relations between different models.

An act $f$ is one-stage if all lotteries $f(s)$ are degenerate; i.e., $f$ assigns outcomes rather than nondegenerate lotteries to all states (upper panel in Fig. 5). Then all relevant uncertainty has been resolved in the first stage. A lottery, identified with the corresponding constant act, is sometimes also called a one-stage lottery (left panel in Fig. 5). Now all relevant uncertainty is resolved in the second stage.

The AA assumptions of EU on $L$ and of monotonicity are called ancillary assumptions. They imply that a function representing preferences over acts must be of the form

$$
V(E U \circ f)
$$

with $V$ nondecreasing. ${ }^{14}$ Further assumptions, in addition to the ancillary assumptions and the common assumptions of weak ordering and nontriviality, concern the function $V$, i.e., the aggregation of uncertainty over $S$, and they concern ambiguity. They are of central interest, and we call them substantive assumptions.

We next discuss the experiment in Section 3 formally. The experiment concerns the two-stage AA model with: (a) $S=\left\{R_{a}, B_{a}\right\}$; (b) bets on the ambiguous urn are acts; (c) bets on the known urn are fifty-fifty lotteries (also some other lotteries are used); (d) $B=10, W=-20$. We used EU to analyze risky choices. We only used a subpart of the two-stage AA model, the rAA model, in two respects. First, all acts and lotteries presented to subjects were one-stage (upper and left panel in Fig. 5). We obtained all desired utility levels at the second stage using consequences that are outcomes, i.e., degenerate lotteries. Yet we could indirectly infer mixtures of consequences under the two-stage AA model if necessary. For example, we could derive, for the AA twin,

$$
\left(R_{a}: 0, B_{a}: 0\right) \sim^{*}\left(R_{a}: 10, B_{a}: 0\right)_{0.5}\left(R_{a}: \alpha, B_{a}: 0\right)=\left(R_{a}:\left(10_{0.5} \alpha\right), B_{a}: 0\right),
$$

\footnotetext{
${ }^{14} \mathrm{We}$ first apply monotonicity with indifferences to show that the representing function is a function $V$ of $E U \circ f$, and then monotonicity in full force to show that $V$ is nondecreasing.
} 


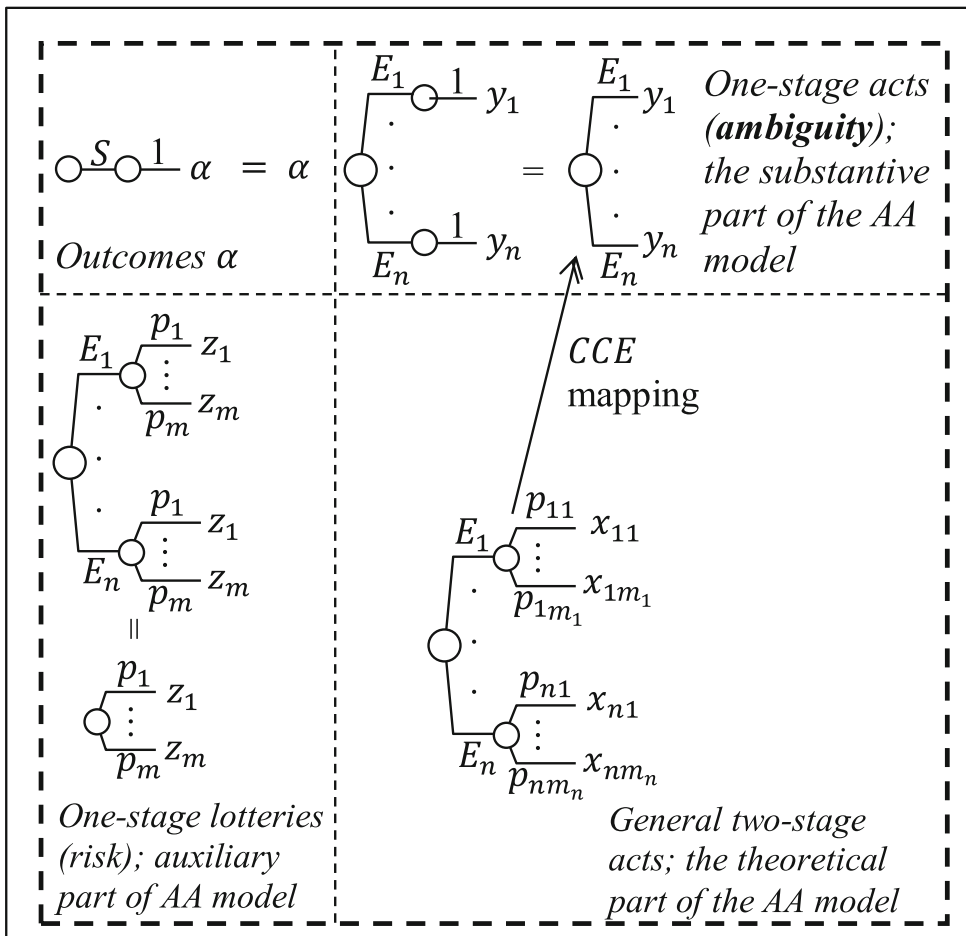

The $C C E$ mapping (value $y_{i}$ for each $i$ ) is defined using the indifferences
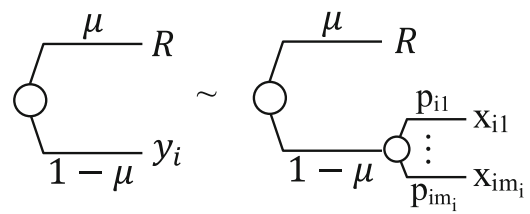

The reduced AA model only concerns the upper (ambiguity) and left (risk) panel, and not the theoretical part. Of the risk panel we only use the subpart with a $\mu$ probability of lottery $R$, to stay away from the upper corner. Only this reduced model is used for empirical work, avoiding the extensive empirical violations in the theoretical part. Through the $C C E$ mapping, the reduced AA model uniquely determines the theoretical part. Thus all theoretical features of the AA model, including its mixture operation, can be used, and all AA decision theories can be tested and applied empirically.

Fig. 5 The reduced AA model 
because we elicited $C E\left(10_{0.5} \alpha\right)=0$. The second respect in which we only used a subpart of the two-stage AA model is that we never used degenerate lotteries when eliciting risky preferences. We next formalize our reduced form of the AA model, called the rAA model.

The first modification [focusing on one-stage acts and lotteries]. For each twostage act $f$, each lottery $f(s)$ is replaced by $C E(f(s))$ (arrow in Fig. 5). This replacement is not done by the decision maker, but by the researcher. We inferred Eq. 18 this way.

The rAA model preserves the underlying mixing of outcomes of the two-stage AA model by temporarily returning to the underlying lotteries for a mix. We use a notation $\beta=\alpha^{\prime} p \gamma=p \alpha+{ }^{\prime}(1-p) \gamma$, where the first prime preceding the subscript probability $p$ indicates that the mixture operation here is different from the original (probabilistic) one in the two-stage AA model, and the second prime following the + sign indicates the same. The two mixture operations are isomorphic though, and lead to the same indifference class. Informally, we take any lotteries $x, z$ with $\alpha=$ $C E(x), \gamma=C E(z)$, we take $y=x_{p} z$, and then get $\beta$ as $C E(y)$. Because of EU on $L$, it does not matter which $x$ and $z$ we take in this process, and the operation is well defined. Always

$$
\beta=\alpha^{\prime} p \gamma=p \alpha+^{\prime}(1-p) \gamma=u^{-1}(p u(\alpha)+(1-p) u(\gamma)) .
$$

This holds irrespective of the particular choices $x$ and $z$. We use Eq. 19 as the formal definition of the new mixture operation. The mixture operation is most easily observable from:

$$
C E\left(\alpha_{p} \gamma\right)=\alpha^{\prime} p \gamma
$$

That is, we take $x=\alpha$ and $z=\gamma$. An example is Eq. 2 which showed that $0=$ $10^{\prime} 0.5 \alpha$.

The second modification [avoiding degenerate lotteries for risky preferences]. In the first modification, we put deterministic outcomes central for the analysis of ambiguity by focusing on one-stage acts. Violations of SEU for such acts, due to ambiguity, are our substantive interest. In the second modification considered now, concerning the analysis of risk through one-stage lotteries, we avoid degenerate lotteries, staying away from the upper left box in Fig. 5 .

We define $R=B_{0.5} W$, and take some fixed $0<\mu<1$ (0.4 in Section 3). For each lottery $x$, we define $x^{\prime}=R_{\mu} x$. Under the ancillary assumptions of the AA model, EU holds on $L$, and then a CE-indifference $\beta \sim y$ is not affected if we bring in $\mu R$, as in

$$
R_{\mu} \beta \sim R_{\mu} y \text {, i.e., } \beta^{\prime} \sim y^{\prime} .
$$

In general, indifferences are not affected under EU if we add or remove primes from all the lotteries. We call $\beta$ in Eq. 21 the conditional $C E$ of $y$, denoted $\beta=C A(y)$. We used this procedure in Eqs. 2 and 3. The $C A$ condition means that there exists a unique CA for each lottery $x \in L$. Given existence, uniqueness can always be achieved by collapsing indifference classes. ${ }^{15}$

\footnotetext{
${ }^{15}$ Skiadas (2013) restricted the AA model to a fixed $n$-tuple of probabilities. If these all exceed $\mu$ and if constant roulette lotteries are excluded, then our second modification is satisfied.
} 
The two modifications combined. The rAA model results from combining the two modifications. Every two-stage act $f$ in the two-stage AA model is replaced by $C A(f)$, defined by replacing every $f(s)$ by $C A(f(s))$, and turning every twostage act into an equivalent one-stage act (arrow in Fig. 5). We carried out the first modification, but with primes added to Eq. 20 because of the second modification.

We call the rAA model derived from the two-stage AA model as just described the corresponding rAA model. Conversely, from every rAA model the uniquely determined corresponding two-stage AA model can be recovered, mostly by deriving preferences between two-stage acts from their $C A$ images. We summarize the rAA model formally.

Definition 5 The reduced $A A(r A A)$ model holds if the following definitions and conditions are satisfied. Assume $S, D, L$ as before. $B$ and $W$ are the best and worst outcomes, $R=W_{0.5} B$, and $0<\mu<1$ is fixed. For each lottery $x$, we have $x^{\prime}=$ $R_{\mu} x$, and $L^{\prime} \subset L$ is the set of all lotteries $x^{\prime} . \mathcal{O} \mathcal{A}$ contains (a) all one-stage acts, and (b) $L^{\prime}$. Thus, all elements of $\mathcal{O} \mathcal{A}$ are one-stage. Preferences $\succcurlyeq$ are defined only over $\mathcal{O} \mathcal{A}$. Preferences over $L^{\prime}$ are represented by EU, the expectation $u$ of a function on $D$ also denoted $u$. Preferences over outcomes agree with those over constant acts, and are represented by $u$ on $D$. Monotonicity holds. Conditional certainty equivalents, denoted CA, are defined as in Eq. 21, and are assumed to uniquely exist for every $x \in L$ (the CA condition). ${ }^{16}$

The mixture operation on outcomes is defined through Eq. 19, and can, for instance, be revealed from indifferences through the following analog of Eq. 20:

$$
C A\left(\alpha_{p} \gamma\right)=\alpha^{\prime} p \gamma \text {. }
$$

We summarize some useful relations between the corresponding reduced and twostage AA models.

Observation 6 Assume the Richness Assumption 1. Then there is a one-to-one correspondence between two-stage and reduced AA models (based on the maps $f \rightarrow C A(f)$ and $\left.x \rightarrow x^{\prime}\right)$, and the preferences of one model uniquely determine those of the other. The rAA model is a substructure of the corresponding two-stage $A A$ model, and its preferences agree with the restriction of the two-stage AA model preferences.

\section{References}

Abdellaoui, M., Vossmann, F., Weber, M. (2005). Choice-based elicitation and decomposition of decision weights for gains and losses under uncertainty. Management Science, 51, 1384-1399.

Abdellaoui, M., Bleichrodt, H., Paraschiv, C. (2007). Loss aversion under prospect theory: A parameterfree measurement. Management Science, 53, 1659-1674.

\footnotetext{
${ }^{16}$ We can and do include all lotteries from $L$ in this definition, also those not contained in $L^{\prime}$, because all preferences only involve elements of $L^{\prime}$.
} 
Abdellaoui, M., Bleichrodt, H., l'Haridon, O., van Dolder, D. (2016). Measuring loss aversion under ambiguity: A method to make prospect theory completely observable. Journal of Risk and Uncertainty, 52, $1-20$.

Ahn, D.S., Choi, S., Gale, D., Kariv, S. (2014). Estimating ambiguity aversion in a portfolio choice experiment. Quantitative Economics, 5, 195-223.

Allais, M. (1953). Fondements d'une Théorie Positive des Choix Comportant un Risque et Critique des Postulats et Axiomes de l'Ecole Américaine. Colloques Internationaux du Centre National de la Recherche Scientifique (Econométrie), 40, 257-332. Paris: Centre National de la Recherche Scientifique. Translated into English, with additions, as The Foundations of a Positive Theory of Choice Involving Risk and a Criticism of the Postulates and Axioms of the American School, in M. Allais \& O. Hagen (1979, Eds.). Expected Utility Hypotheses and the Allais Paradox, 27-145. Reidel, Dordrecht, the Netherlands.

Anscombe, F.J., \& Aumann, R.J. (1963). A definition of subjective probability. Annals of Mathematical Statistics, 34, 199-205.

Baillon, A., \& Bleichrodt, H. (2015). Testing ambiguity models through the measurement of probabilities for gains and losses. American Economic Journal: Microeconomics, 7, 77-100.

Baillon, A., \& Placido, L. (2017). Testing constant absolute and relative ambiguity aversion. Working paper.

Baltussen, G., van den Assem, M.J., van Dolder, D. (2016). Risky choice in the limelight. Review of Economics and Statistics, 98, 318-332.

Baucells, M., \& Villasis, A. (2015). Equal tails: A simple method to elicit utility under violations of expected utility. Decision Analysis, 12, 190-204.

Birnbaum, M.H. (2008). New paradoxes of risky decision making. Psychological Review, 115, 463-501.

Bleichrodt, H., \& Miyamoto, J. (2003). A characterization of quality-adjusted life-years under cumulative prospect theory. Mathematics of Operations Research, 28, 181-193.

Bleichrodt, H., Schmidt, U., Zank, H. (2009). Additive utility in prospect theory. Management Science, $55,863-873$.

Bommier, A. (2017). A dual approach to ambiguity aversion. Journal of Mathematical Economics, 71, 104-118.

Bommier, A., Kochov, A., le Grand, F. (2017). On monotone recursive preferences. Econometrica, 85, 1433-1466.

Borah, A., \& Kops, C. (2016). The Anscombe-Aumann representation and the independence axiom: A reconsideration. Theory and Decision, 80, 211-226.

Bruhin, A., Fehr-Duda, H., Epper, T. (2010). Risk and rationality: Uncovering heterogeneity in probability distortion. Econometrica, 78, 1375-1412.

Camerer, C.F., \& Weber, M. (1992). Recent developments in modelling preferences: Uncertainty and ambiguity. Journal of Risk and Uncertainty, 5, 325-370.

Cerreia-Vioglio, S., Ghirardato, P., Maccheroni, F., Marinacci, M., Siniscalchi, M. (2011). Rational preferences under ambiguity. Economic Theory, 48, 341-375.

Cerreia-Vioglio, S., Maccheroni, F., Marinacci, M. (2015). Put-call parity and market frictions. Journal of Economic Theory, 157, 730-762.

Chambers, R.G., Grant, S., Polak, B., Quiggin, J. (2014). A two-parameter model of dispersion aversion. Journal of Economic Theory, 150, 611-641.

Chateauneuf, A. (1991). On the use of capacities in modeling uncertainty aversion and risk aversion. Journal of Mathematical Economics, 20, 343-369.

Chateauneuf, A., Eichberger, J., Grant, S. (2007). Choice under uncertainty with the best and worst in mind: NEO-additive capacities. Journal of Economic Theory, 137, 538-567.

Chateauneuf, A., \& Faro, J. (2009). Ambiguity through confidence functions. Journal of Mathematical Economics, 45, 535-558.

Cheridito, P., Delbaen, F., Drapeau, S., Kupper, M. (2015). Stochastic order-monotone uncertainty-averse preferences. Working paper.

Chew, S.H., Li, K.K., Chark, R., Zhong, S. (2008). Source preference and ambiguity aversion: Models and evidence from behavioral and neuroimaging experiments. In Houser, D., \& McGabe, K. (Eds.) Neuroeconomics. Advances in health economics and health services research (Vol. 20, pp. 179-201). Bingley: JAI Press.

Choi, S., Fishman, R., Gale, D., Kariv, S. (2007). Consistency and heterogeneity of individual behavior under uncertainty. American Economic Review, 97, 1921-1938. 
Cubitt, P.R., Starmer, C., Sugden, R. (1998). Dynamic choice and the common ratio effect: An experimental investigation. Economic Journal, 108, 1362-1380.

de Finetti, B. (1937). La prévision: Ses lois logiques, ses sources subjectives. Annales de l'Institut Henri Poincaré, 7, 1-68. Translated into English by Henry E. Kyburg, Foresight: Its Logical Laws, its Subjective Sources. In H. E. Kyburg and H. E. Smokler (1964, Eds). Studies in Subjective Probability. Wiley, New York, 53-118; 2nd edition 1980, Krieger, New York.

de Lara Resende, J.G., \& Wu, G. (2010). Competence effects for choices involving gains and losses. Journal of Risk and Uncertainty, 40, 109-132.

Dean, M., \& Ortoleva, P. (2017). Allais, Ellsberg, and preferences for hedging. Theoretical Economics, $12,377-424$.

Dimmock, S.G., Kouwenberg, R., Mitchell, O.S., Peijnenburg, K. (2015). Estimating ambiguity preferences and perceptions in multiple prior models: Evidence from the field. Journal of Risk and Uncertainty, 51, 219-244.

Dobbs, I.M. (1991). A Bayesian approach to decision-making under ambiguity. Economica, 58, 417-440.

Dominiak, A., \& Lefort, J.-P. (2011). Unambiguous events and dynamic Choquet preferences. Economic Theory, 46, 401-425.

Dominiak, A., \& Schnedler, W. (2011). Attitudes toward uncertainty and randomization: An experimental study. Economic Theory, 48, 289-312.

Dominiak, A., Duersch, P., Lefort, J.-P. (2012). A dynamic Ellsberg urn experiment. Games and Economic Behavior, 75, 625-638.

Du, N., \& Budescu, D. (2005). The effects of imprecise probabilities and outcomes in evaluating investment options. Management Science, 51, 1791-1803.

Edwards, W. (1954). The theory of decision making. Psychological Bulletin, 51, 380-417.

Eichberger, J., \& Kelsey, D. (1996). Uncertainty aversion and dynamic consistency. International Economic Review, 37, 625-640.

Ellsberg, D. (1961). Risk, ambiguity and the Savage axioms. Quarterly Journal of Economics, 75, 643669.

Faro, J.H. (2005). On the choices under ambiguity. Ph.D. dissertation. Instituto Nacional de Matemática Pura e Aplicada, Rio de Janeiro.

Fehr-Duda, H., \& Epper, T. (2012). Probability and risk: Foundations and economic implications of probability-dependent risk preferences. Annual Review of Economics, 4, 567-593.

Fox, C.R., Erner, C., Walters, D.J. (2015). Decision under risk: From the field to the laboratory and back. In Keren, G., \& Wu, G. (Eds.) The Wiley Blackwell handbook of judgment and decision making (pp. 4388). Oxford: Blackwell.

Gajdos, T., Hayashi, T., Tallon, J.-M., Vergnaud, J.-C. (2008). Attitude towards imprecise information. Journal of Economic Theory, 140, 27-65.

Ghirardato, P., \& Marinacci, M. (2001). Risk, ambiguity, and the separation of utility and beliefs. Mathematics of Operations Research, 26, 864-890.

Ghirardato, P., Maccheroni, F., Marinacci, M. (2004). Differentiating ambiguity and ambiguity attitude. Journal of Economic Theory, 118, 133-173.

Gilboa, I. (1987). Expected utility with purely subjective non-additive probabilities. Journal of Mathematical Economics, 16, 65-88.

Gilboa, I., \& Schmeidler, D. (1989). Maxmin expected utility with a non-unique prior. Journal of Mathematical Economics, 18, 141-153.

Grant, S., \& Polak, B. (2013). Mean-dispersion preferences and constant absolute uncertainty aversion. Journal of Economic Theory, 148, 1361-1398.

Hayashi, T., \& Miao, J. (2011). Intertemporal substitution and recursive smooth ambiguity preferences. Theoretical Economics, 6, 423-475.

Jaffray, J.-Y. (1994). Dynamic decision making with belief functions. In Yager, R.R., Fedrizzi, M., Kacprzyk, J. (Eds.) Advances in the Dempster-Shafer theory of evidence (pp. 331-352). New York: Wiley.

Kahneman, D., \& Tversky, A. (1975). Value theory: An analysis of choices under risk. Paper presented at a conference on public economics. Jerusalem 1975.

Kahneman, D., \& Tversky, A. (1979). Prospect theory: An analysis of decision under risk. Econometrica, 47, 263-291.

Karni, E., \& Schmeidler, D. (1991). Atemporal dynamic consistency and expected utility theory. Journal of Economic Theory, 54, 401-408. 
Keynes, J.M. (1921). A treatise on probability. London: McMillan.

Klibanoff, P., Marinacci, M., Mukerji, S. (2005). A smooth model of decision making under ambiguity. Econometrica, 73, 1849-1892.

Knight, F.H. (1921). Risk, uncertainty, and profit. New York: Houghton Mifflin.

Köbberling, V., \& Wakker, P.P. (2003). Preference foundations for nonexpected utility: A generalized and simplified technique. Mathematics of Operations Research, 28, 395-423.

Kocher, M.G., Lahno, A.M., Trautmann, S.T. (2018). Ambiguity aversion is not universal. European Economic Review, 101, 268-283.

Kopylov, I. (2009). Choice deferral and ambiguity aversion. Theoretical Economics, 4, 199-225.

Köszegi, B., \& Rabin, M. (2006). A model of reference-dependent preferences. Quarterly Journal of Economics, 121, 1133-1165.

Kothiyal, A., Spinu, V., Wakker, P.P. (2011). Prospect theory for continuous distributions: A preference foundation. Journal of Risk and Uncertainty, 42, 195-210.

Kreps, D.M. (1988). Notes on the theory of choice. Boulder: Westview Press.

Luce, R.D. (2000). Utility of gains and losses: Measurement-theoretical and experimental approaches. London: Lawrence Erlbaum Publishers.

Maccheroni, F., Marinacci, M., Rustichini, A. (2006). Ambiguity aversion, robustness, and the variational representation of preferences. Econometrica, 74, 1447-1498.

Machina, M.J. (1989). Dynamic consistency and non-expected utility models of choice under uncertainty. Journal of Economic Literature, 27, 1622-1688.

Machina, M.J. (2014). Ambiguity aversion with three or more outcomes. American Economic Review, $104,3814-3840$.

McCord, M.R., \& de Neufville, R. (1986). Lottery equivalents: Reduction of the certainty effect problem in utility assessment. Management Science, 32, 56-60.

Nau, R.F. (2006). Uncertainty aversion with second-order utilities and probabilities. Management Science, $52,136-145$.

Neilson, W.S. (2010). A simplified axiomatic approach to ambiguity aversion. Journal of Risk and Uncertainty, 41, 113-124.

Oechssler, J., Rau, H., Roomets, A. (2016). Hedging and ambiguity. Working paper.

Ozdenoren, E., \& Peck, J. (2008). Ambiguity aversion, games against nature, and dynamic consistency. Games and Economic Behavior, 62, 106-115.

Pulford, B.D. (2009). Is luck on my side? Optimism, pessimism, and ambiguity aversion. Quarterly Journal of Experimental Psychology, 62, 1079-1087.

Ryan, M.J. (2009). Generalizations of SEU: A geometric tour of some non-standard models. Oxford Economic Papers, 61, 327-354.

Saito, K. (2015). Preferences for flexibility and randomization under uncertainty. American Economic Review, 105, 1246-1271.

Saponara, N. (2017). Revealed understanding. Working paper.

Savage, L.J. (1954). The foundations of statistics. New York: Wiley. (Second edition 1972, Dover Publications, New York).

Schmeidler, D. (1989). Subjective probability and expected utility without additivity. Econometrica, 57, 571-587.

Schmidt, U. (2003). Reference-dependence in cumulative prospect theory. Journal of Mathematical Psychology, 47, 122-131.

Schmidt, U. (2004). Alternatives to expected utility: Some formal theories. In Barber, S., Hammond, P. J., Seidl, C. (Eds.) Handbook of utility theory II (Ch. 15, pp. 757-838). Dordrecht: Kluwer Academic Publishers.

Schmidt, U., \& Zank, H. (2009). A simple model of cumulative prospect theory. Journal of Mathematical Economics, 45, 308-319.

Schneider, F., \& Schonger, M. (2017). An experimental test of the Anscombe-Aumann monotonicity axiom. Working paper.

Siniscalchi, M. (2009). Vector expected utility and attitudes toward variation. Econometrica, 77, 801855.

Siniscalchi, M. (2011). Dynamic choice under ambiguity. Theoretical Economics, 6, 379-421.

Skiadas, C. (2013). Scale-invariant uncertainty-averse preferences and source-dependent constant relative risk aversion. Theoretical Economics, 8, 59-93.

Skiadas, C.S. (2015). Dynamic choice with constant source-dependent relative risk aversion. Economic Theory, 60, 393-422. 
Slovic, P., Lichtenstein, S., Fischhoff, B. (1988). Decision making. In Atkinson, R.C., Herrnstein, R.J., Lindzey, G.E., Luce, R.D. (Eds.) Stevens handbook of experimental psychology (Vol. 2, pp. 673-738). New York: Wiley.

Starmer, C. (2000). Developments in non-expected utility theory: The hunt for a descriptive theory of choice under risk. Journal of Economic Literature, 38, 332-382.

Strzalecki, T. (2011). Axiomatic foundations of multiplier preferences. Econometrica, 79, 47-73.

Sugden, R. (2003). Reference-dependent subjective expected utility. Journal of Economic Theory, 111, 172-191.

Trautmann, S.T., \& van de Kuilen, G. (2015). Ambiguity attitudes. In Keren, G., \& Wu, G. (Eds.) The Wiley Blackwell handbook of judgment and decision making (Ch. 3, pp. 89-116). Oxford: Blackwell.

Tversky, A., \& Kahneman, D. (1981). The framing of decisions and the psychology of choice. Science, $211,453-458$.

Tversky, A., \& Kahneman, D. (1992). Advances in prospect theory: Cumulative representation of uncertainty. Journal of Risk and Uncertainty, 5, 297-323.

Viscusi, W.K., \& O'Connor, C. (1984). Adaptive responses to chemical labeling: Are workers Bayesian decision makers? American Economic Review, 74, 942-956.

Viscusi, W.K., \& Evans, W.N. (2006). Behavioral probabilities. Journal of Risk and Uncertainty, 32, 5-15.

Wakker, P.P. (1989). Continuous subjective expected utility with nonadditive probabilities. Journal of Mathematical Economics, 18, 1-27.

Wakker, P.P. (2010). Prospect theory: For risk and ambiguity. Cambridge: Cambridge University Press.

Wakker, P.P., \& Tversky, A. (1993). An axiomatization of cumulative prospect theory. Journal of Risk and Uncertainty, 7, 147-176.

Yechiam, E., Stout, J.C., Busemeyer, J.R., Rock, S.L., Finn, P.R. (2005). Individual differences in the response to forgone payoffs: An examination of high functioning drug abusers. Journal of Behavioral Decision Making, 18, 97-110.

Zeckhauser, R., \& Viscusi, W.K. (1990). Risk within reason. Science, 248(4955), 559-564. 